\title{
Analysis of full-waveform LiDAR data for classification of an orange orchard scene
}

Article

Published Version

Creative Commons: Attribution 3.0 (CC-BY)

Fieber, K. D., Davenport, I. J., Ferryman, J. M., Gurney, R. J., Walker, J. P. and Hacker, J. M. (2013) Analysis of fullwaveform LiDAR data for classification of an orange orchard scene. ISPRS Journal of Photogrammetry and Remote Sensing, 82 (13). pp. 63-82. ISSN 0924-2716 doi: https://doi.org/10.1016/j.isprsjprs.2013.05.002 Available at https://centaur.reading.ac.uk/32918/

It is advisable to refer to the publisher's version if you intend to cite from the work. See Guidance on citing.

To link to this article DOI: http://dx.doi.org/10.1016/j.isprsjprs.2013.05.002

Publisher: Elsevier

All outputs in CentAUR are protected by Intellectual Property Rights law, including copyright law. Copyright and IPR is retained by the creators or other copyright holders. Terms and conditions for use of this material are defined in the End User Agreement.

\section{www.reading.ac.uk/centaur}

\section{CentAUR}


Central Archive at the University of Reading

Reading's research outputs online 


\title{
Analysis of full-waveform LiDAR data for classification of an orange orchard scene ${ }^{\text {is }}$
}

\author{
Karolina D. Fieber ${ }^{\mathrm{a}, *}$, Ian J. Davenport ${ }^{\mathrm{b}}$, James M. Ferryman ${ }^{\mathrm{a}}$, Robert J. Gurney ${ }^{\mathrm{b}}$, Jeffrey P. Walker ${ }^{\mathrm{c}}$, \\ Jorg M. Hacker ${ }^{\mathrm{d}}$ \\ ${ }^{a}$ University of Reading, School of Systems Engineering, Whiteknights, Reading RG6 6AY, UK \\ ${ }^{\mathrm{b}}$ University of Reading, School of Mathematical and Physical Sciences, Harry Pitt Building, Reading RG6 6AL, UK \\ ${ }^{\mathrm{c}}$ Monash University, Faculty of Engineering, Clayton Campus, Melbourne, VIC 3800, Australia \\ d Airborne Research Australia, School of the Environment, Flinders University, Adelaide, SA 5001, Australia
}

\section{A R T I C L E I N F O}

\section{Article history:}

Received 18 December 2012

Received in revised form 29 April 2013

Accepted 2 May 2013

\section{Keywords:}

Full-waveform

LiDAR

Backscattering coefficient

Classification

Reflectance

Vegetation

\begin{abstract}
A B S T R A C T
Full-waveform laser scanning data acquired with a Riegl LMS-Q560 instrument were used to classify an orange orchard into orange trees, grass and ground using waveform parameters alone. Gaussian decomposition was performed on this data capture from the National Airborne Field Experiment in November 2006 using a custom peak-detection procedure and a trust-region-reflective algorithm for fitting Gauss functions. Calibration was carried out using waveforms returned from a road surface, and the backscattering coefficient $\gamma$ was derived for every waveform peak. The processed data were then analysed according to the number of returns detected within each waveform and classified into three classes based on pulse width and $\gamma$. For single-peak waveforms the scatterplot of $\gamma$ versus pulse width was used to distinguish between ground, grass and orange trees. In the case of multiple returns, the relationship between first (or first plus middle) and last return $\gamma$ values was used to separate ground from other targets. Refinement of this classification, and further sub-classification into grass and orange trees was performed using the $\gamma$ versus pulse width scatterplots of last returns. In all cases the separation was carried out using a decision tree with empirical relationships between the waveform parameters. Ground points were successfully separated from orange tree points. The most difficult class to separate and verify was grass, but those points in general corresponded well with the grass areas identified in the aerial photography. The overall accuracy reached $91 \%$, using photography and relative elevation as ground truth. The overall accuracy for two classes, orange tree and combined class of grass and ground, yielded $95 \%$. Finally, the backscattering coefficient $\gamma$ of single-peak waveforms was also used to derive reflectance values of the three classes. The reflectance of the orange tree class (0.31) and ground class (0.60) are consistent with published values at the wavelength of the Riegl scanner $(1550 \mathrm{~nm})$. The grass class reflectance $(0.46)$ falls in between the other two classes as might be expected, as this class has a mixture of the contributions of both vegetation and ground reflectance properties.
\end{abstract}

(c) 2013 The authors. Published by Elsevier B.V. All rights reserved.

\section{Introduction}

\subsection{Motivation}

Land cover classification is being used for a range of applications, including the estimation of biomass, calculation of carbon stocks, and identification of land use change. However, such classifications have largely relied upon multispectral aerial and satellite imagery to date. A limitation of this approach is that it does not

\footnotetext{
This is an open-access article distributed under the terms of the Creative Commons Attribution License, which permits unrestricted use, distribution, and reproduction in any medium, provided the original author and source are credited.

* Corresponding author. Tel.: +44 (0)118 378 7633; fax: +44 1189751994.

E-mail address: k.fieber@pgr.reading.ac.uk (K.D. Fieber).
}

provide the structure of flora or information on the sub-canopy because the instrument field-of-view often captures only the wellilluminated upper canopy. With a footprint diameter of around $25 \mathrm{~cm}$, and use of active measurement techniques that can detect returns from a small fraction of that footprint, a laser beam has the potential to penetrate deep into a vegetated landscape. Furthermore by sending several beams per square metre, laser altimetry should therefore be capable of providing detailed structural information about the vegetation by analysing the shape of the light curve returned.

\subsection{Background}

Small footprint full-waveform airborne LiDAR data have become increasingly available in recent years (Mallet et al., 2011). 
In comparison to so-called discrete systems, full-waveform scanners offer additional information about the targets included in the footprint than location alone (Mallet and Bretar, 2009). This extra information is derived from (i) peak amplitude which relates to radiometric properties of the target and (ii) pulse width, which is a measure of surface roughness and slope. Full-waveform systems also give the user more flexibility in comparison to discrete systems, as the processing stage is performed off-line and allows for adjustment of the processing methods to match the application.

The development of full-waveform scanning instruments has brought interest in the suitability of additional features offered by those systems for many applications. One of the obvious areas for testing the relevance of the new features is classification. Several studies have been carried out on benefits of full-waveform features for classification, both in the built-up environment as well as forested/vegetated areas. Nevertheless, even if classification is performed in an urban environment, it is usually limited to binary detection of urban vegetation due to complex nature of those sites (Ducic et al., 2006; Gross et al., 2007; Höfle and Hollaus, 2010; Höfle et al., 2012; Rutzinger et al., 2008; Wagner et al., 2008). Ducic et al. (2006) explored the histograms of amplitude and width of four urban classes. However, due to significant overlap of four classes in amplitude-width space, they were able to perform only binary vegetation/non-vegetation classification with $88.6 \%$ accuracy. Wagner et al. (2008) also carried out vegetation/non-vegetation classification achieving 93.7\% accuracy for a baroque garden. A number of studies distinguish more than vegetation and non-vegetation classes in an urban environment including Mallet et al. (2008, 2011), Alexander et al. (2010), Guo et al. (2011). The first two studies managed to separate ground, vegetation and buildings with 92\% and 95\% accuracy, respectively. Guo et al. (2011) distinguished between four classes, namely building, vegetation, artificial and natural ground, achieving an accuracy of 95\%. Similarly, Alexander et al. (2010) separated six classes: trees, shrubs, grass, road, flat and pitched roofs with $92 \%$ overall accuracy.

The benefits of full-waveform laser scanning data are particularly profound for forestry, because such data provide detailed vertical structure of vegetation as well as their radiometric properties. This in turn can provide better estimation of above ground biomass and stem volume, and better classification. Several promising studies have been carried out in forest environment with the emphasis on species, mostly deciduous versus coniferous classification (Heinzel and Koch, 2011; Höfle et al., 2008; Litkey et al., 2007; Reitberger et al., 2006, 2008; Yao et al., 2012). Preliminary studies by Reitberger et al. (2006) and Litkey et al. (2007), showed the benefits of waveform data over discrete laser scanning for distinguishing tree species (deciduous and coniferous) based only on increased number of extracted points. Furthermore, both authors noticed higher point density in the upper canopy in deciduous trees. Reitberger et al. (2008) extended the number of tested features (including features describing outer and internal geometry of the trees, intensity and width features, and number of reflections) and performed classification of deciduous and coniferous trees under leaf-off and leaf-on conditions with 95\% and 85\% accuracy, respectively. Höfle et al. (2008), who did not perform classification, explored the potential of segment based classification of tree species using only amplitude and width. Heinzel and Koch (2011) classified six species with $57 \%$ accuracy, four species with $78 \%$ accuracy, and decidous/coniferous trees with $91 \%$ accuracy using the statistics of full-waveform derived parameters. Neuenschwander et al. (2009) separated seven vegetation classes with $86 \%$ classification accuracy, including two tree species, dead trees, grass and bushes.

\subsection{Review of related work}

\subsubsection{Waveform processing}

Much has been published on methods of LiDAR waveform processing for extraction of additional parameters and their applications. Hofton et al. (2000) proposed a waveform processing technique called Gaussian decomposition, that uses a LevenbergMarquardt optimisation algorithm for fitting large footprint (25 m) waveforms with Gaussian functions. Wagner et al. (2006) adjusted this method to small footprint LiDAR data and presented a theoretical background for it. Other methods of waveform processing have also been proposed including the Averaged Square Difference Function (ASDF) method presented by Roncat et al. (2008) and the reversible jump Markov chain Monte Carlo (RJMCMC) proposed by Hernandez-Marin et al. (2007). Gaussian decomposition is, nevertheless, the most often used and the most established method of waveform parameter extraction. Several modifications of this method have been presented, including different ways of initial parameter estimation or different fitting algorithms. Persson et al. (2005) proposed to use an Expectation Maximisation algorithm for waveform decomposition while Lin et al. (2010) developed the Rigorous Pulse Detection method which allows for weak pulse detection, and uses a Trust Region algorithm for fitting functions. Chauve et al. (2007) suggested using log-normal and generalized Gaussian functions for fitting in order to account for asymmetric, flattened and peaked pulses. This method was further extended by Mallet et al. (2009) who added additional fitting functions to the library such as Burr, Weibull and Nakagami. Some authors (Neuenschwander et al., 2009; Zhu et al., 2011) fit the functions to individual peaks and subsequently subtract them from the waveform to find the remaining peaks while others perform the fitting on all peaks simultaneously (Lin et al., 2010; Wagner et al., 2006).

Together with processing techniques, radiometric calibration of the data has been addressed by several authors. Wagner et al. (2006) proposed calibration using asphalt road and its estimated reflectance (0.2 at $1550 \mathrm{~nm}$ for Riegl LMS-Q560), and suggested using backscatter cross-section as the calibrated parameter of each peak. Briese et al. (2008) improved this method by proposing measurement of the natural target's reflectance in the field using a reflectometer and by excluding the atmospheric transmission factor from the calibration constant. A similar procedure was followed by Höfle et al. (2008), however, in this study atmospheric attenuation effects were included in the calibration constant. Wagner (2010) presented an extended theoretical background for LiDAR calibration and recommended using the backscattering coefficient $\gamma$ (backscatter cross-section normalized by the footprint) in place of backscatter cross-section. Wagner (2010) also suggested taking amplitude variations of the transmitted pulse into account, thereby excluding amplitude of the system waveform from the calibration constant. Finally, Roncat et al. (2011) examined the influence of width and amplitude of transmitted pulse on variations of calibration constant, and concluded that variation of transmitted amplitude can be regarded as the main influence while the width of transmitted pulse can be neglected. In summary, decomposition of waveforms and calibration of LiDAR data have been widely studied and are fairly established areas of research.

\subsubsection{Classification methods}

Classification of LiDAR data can be performed on a per point basis, when each XYZ point is attributed with a set of features (Alexander et al., 2010; Bretar et al., 2009; Ducic et al., 2006; Mallet et al., 2011, 2008; Wagner et al., 2008). Another approach is to rasterise waveform features to cope more efficiently with the large amount of data (Guo et al., 2011; Heinzel and Koch, 2011; Neuenschwander et al., 2009). Finally, the data can be segmented first, and subsequently a set of features attached to each of the segments 
(Höfle and Hollaus, 2010; Höfle et al., 2012, 2008; Reitberger et al., 2008; Rutzinger et al., 2008) or computed for an neighbourhood volumetric environment (Gross et al., 2007). Several approaches have been developed to segment the data prior to classification. Segmentation of vegetation in urban scene data was performed by Rutzinger et al. (2008) based on echo width homogeneity using a seeded region growing approach. Conversely, Höfle et al. (2008), segmented forest data using an edge based procedure on a normalized digital surface model (also called canopy height model) that delineates convex elevated objects. This segmentation procedure was then adapted for urban vegetation detection by Höfle and Hollaus (2010) and Höfle et al. (2012). A different segmentation approach is shown in Reitberger et al. (2008), who performed segmentation using a watershed algorithm applied to a smoothed canopy height model in forested areas. Reitberger et al. (2009) enhanced the watershed segmentation procedure by adding a special stem detection method and three-dimensional segmentation of single trees using a normalized cut method. This approach proved successful in detecting small trees in the lower parts of the forest, and improved single tree detection by $12 \%$ in comparison to watershed segmentation.

Several classification methods have been employed in the literature using full-waveform data. This includes rule based methods such as decision trees or simple thresholds both set up manually (Ducic et al., 2006; Gross et al., 2007; Höfle and Hollaus, 2010; Wagner et al., 2008) and automatically (Alexander et al., 2010; Höfle et al., 2012; Rutzinger et al., 2008). The advantage of the decision tree method is such that it requires no assumptions in terms of data distribution, and its linear flow makes it straightforward to interpret. Other methods use classifiers based on statistical learning and are usually more difficult to understand. Support Vector Machine was used by Mallet et al. $(2008,2011)$ and Bretar et al. (2009). This method is not dependant on data dimensionality thus it is well suited for multi source and high dimensional problems with limited training sets. It belongs to non-parametric methods and performs non-linear classification. A Random Forest algorithm, employed in Guo et al. (2011), is also suited for multi source and large datasets and moreover it provides information about feature importance. An artificial neural network classifier, evaluated in Höfle et al. (2012) is a method that imitates the way the human brain works. It is, therefore, very difficult to understand the complexity of the interactions between the algorithm's neurons. A Bayesian pair-wise classifier, used by Neuenschwander et al. (2009) allows evaluation of feature performance by pair-wise comparison. This method provides the most discriminative class dependant features by incremental rating of their contributions. Supervised classifiers are usually preferred by authors, nevertheless some of them perform unsupervised classification (Reitberger et al., 2008). Yao et al. (2012) compared unsupervised (Expectation-Maximisation) and supervised (Maximum Likelihood) classification; the latter was found to yield a slightly higher overall accuracy (by $2 \%$ ).

Different approaches to the full-waveform LiDAR classification have been studied. Some of the studies consider only geometrical features and benefit only from denser point clouds (Reitberger et al., 2006; Litkey et al., 2007). Others explore the additional value of width and (uncalibrated) amplitude (or intensity) parameters extracted in the process of decomposition, together with geometrical features (Bretar et al., 2009; Gross et al., 2007; Heinzel and Koch, 2011; Mallet et al., 2008; Neuenschwander et al., 2009; Reitberger et al., 2008; Rutzinger et al., 2008). Now that calibration procedures are well established, more studies use calibrated parameters such as backscatter cross-section and backscattering coefficient in place of amplitude, or as additional features for classification (Alexander et al., 2010; Guo et al., 2011; Höfle et al., 2012, 2008; Mallet et al., 2011; Wagner et al., 2008). An original approach presented by Neuenschwander et al. (2008) analysed the potential of structure-based parameters (raw-waveform de- rived) such as rise time to the first peak, canopy height, ratio of canopy to ground energy, and total integrated energy of the entire waveform for classification of nine urban classes, but did not perform the classification itself. This approach was explored further for classification of seven vegetation classes in a woodland scene by Neuenschwander et al. (2009), and it outperformed Quickbird classification by $15 \%$ reaching $85.8 \%$ accuracy.

\subsubsection{Contribution of waveform parameters}

Several authors have also carried out studies to measure the contribution of full-waveform features against other features that could be extracted from discrete LiDAR systems, and attempted to determine the most discriminative ones. Mallet et al. (2011) and Guo et al. (2011) presented extensive studies of parameters contributing to better accuracy of urban scene classification. Mallet et al. (2011) tested 27 parameters altogether including geometric and full-waveform LiDAR features for classification of three classes. Guo et al. (2011) assessed 12 features being a combination of multispectral and LiDAR input to classify four classes. Both studies found the height difference in a neighbourhood environment between the point of interest and the lowest point as the most discriminative feature. Among full-waveform parameters, both authors reported amplitude (corrected in Mallet et al. (2011)) and backscatter cross-section to contribute significantly to high accuracies (in both cases 95\%). To this list, Mallet et al. (2011) added backscattering coefficient, which was not tested in Guo et al. (2011), who in turn found two multispectral features (red and blue band) highly discriminative. The value of features was also analysed on a per class basis in Guo et al. (2011), finding some features to be class specific (e.g. normalized echo number, etc.). The relevance of full-waveform parameters was also confirmed by comparison with performance of discrete LiDAR parameters. Although the overall accuracy was similar (93\% and 92\% respectively), discrete LiDAR features completely failed to separate natural ground from artificial ground. Nevertheless, a similar comparison in Mallet et al. (2011) showed a much lower average accuracy for full-waveform derived features (64.8\%) as compared to discrete features (91.4\%). However, in both cases the combination of both types of features improved classification accuracy by up to $3 \%$. A comparison of the performance of amplitude, backscatter cross-section and backscattering coefficient in combination with other geometric features for classification of six classes in urban area was carried out by Alexander et al. (2010). The highest accuracy was achieved for the dataset that included backscattering coefficient (92\%) whereas amplitude and backscatter cross-section yielded only $74 \%$ accuracy.

Some full-waveform LiDAR classification studies use echo ratio relationships, such as the number of first and middle returns over the number of single returns (Rutzinger et al., 2008), or number of first and middle returns over the number of single and last returns (Höfle and Hollaus, 2010; Höfle et al., 2012, 2008), or the number of single returns over the number of multiple returns (Reitberger et al., 2008). However, few so far (Heinzel and Koch, 2011; Höfle and Hollaus, 2010; Höfle et al., 2012; Reitberger et al., 2008; Wagner et al., 2008) have taken into account to some extent the potential different properties of peaks (i.e. amplitude, backscatter crosssection or backscattering coefficient) according to the number of targets included in the footprint. Höfle and Hollaus (2010) and Höfle et al. (2012) calculated features for all, first, first and middle, and combined last and single echoes, however did not analyse the single and last returns separately. Reitberger et al. (2008) used mean pulse width of single and first reflections whereas Heinzel and Koch (2011) used different features calculated for all, first and single echoes. Wagner et al. (2008) presented differences in backscatter cross-section in relation to peaks in single-, two- and three-peak waveforms. 


\subsection{Objectives of this work}

This paper seeks to show the potential of calibrated full-waveform features for classification of a rural scene without the use of any geometrical or neighbourhood relations. The hypothesis is that calibrated full-waveform return features (width and backscattering coefficient) alone can be used for classification purposes with high accuracy results. At the same time, the emphasis is placed on the fact the analysis should be carried out separately for single and multiple-return waveforms due to their different characteristics especially in terms of backscattering coefficient. Consequently, this study, through exploratory data analysis, investigates fullwaveform LiDAR data parameters according to the number of returns occurring in the waveform train. This approach seems relevant as the waveform parameters tend to vary depending on the number of components in the waveform as well as their position within the train. Single-peak waveforms deserve special attention. Since they represent extended targets, their parameters can be directly related to the target's radiometric properties.

The rest of this paper is organised as follows. The data and the study area are described in Section 2. Gaussian decomposition is performed using a dedicated procedure (Section 3.1) with a Trust Region Reflective optimisation algorithm, as opposed to some studies that use data processed by commercial Riegl software RiANALYZE (Alexander et al., 2010; Heinzel and Koch, 2011; Höfle and Hollaus, 2010; Höfle et al., 2012, 2008). This procedure allows for the detection of weak echoes and provides a more complete description of the tree canopy in comparison to RiANALYZE. Furthermore, the data is calibrated using an asphalt road, taking into account variability in the transmitted pulse. The backscattering coefficient is then derived for each data point. Exploratory data analysis of width and backscattering coefficient of single and last returns is carried out. Subsequently, per-point classification of three classes, namely ground, orange trees and grass is performed. Similarly to Ducic et al. (2006) and Wagner et al. (2008) classification is conducted using solely waveform parameters and rulebased decision tree (Section 3.5). The quality of classification is then assessed with the use of relative elevation, and the combination of elevation and aerial photography (Sections 4.1 and 4.2). Finally, an attempt to estimate the reflectance values of the three classes is made in Section 4.3. Conclusions and further work are given in Section 5.

\section{Study area and data}

\subsection{Study area}

The data used in this study was acquired as part of National Airborne Field Experiment (NAFE) carried out in Australia in November 2006. The aim of the campaign was to map near-surface soil moisture at a range of resolutions making use of passive microwave airborne and space-borne remote sensors and to test the suitability of the site for Soil Moisture and Ocean Salinity (SMOS) calibration (Merlin et al., 2008; Walker et al., 2006).

The study area is located near the township of Yanco in southern New South Wales, Australia, within the Murrumbidgee catchment. An area of $150 \mathrm{~m}$ by $80 \mathrm{~m}$ of orange orchard located between 55,393,360 $\mathrm{m}$ and 55,393,510 $\mathrm{m}$ (Easting) and between $6,169,250 \mathrm{~m}$ and $6,169,330 \mathrm{~m}$ (Northing) (UTM, zone $55 \mathrm{H}$ ) was selected as the test site for this study (Fig. 1). Ground elevation ranges from $122 \mathrm{~m}$ to $126 \mathrm{~m}$ across the site, with the lowest elevations in the North West corner and rising towards the South. The orange trees are denser and taller in the South East while being small and sparse in the North West. The direction of orange tree rows is South West to North East (at about $60^{\circ}$ angle from the
North) and the rows are about $7 \mathrm{~m}$ apart. Soils in the Murrumbidgee vary from sandy to clayey (Walker et al., 2006) with loams, composed of sand, silt and clay dominating in the Yanco area (Monerris et al., 2011).

\subsection{LiDAR data}

The laser scanning data was acquired by Airborne Research Australia on November 3rd 2006 with a full-waveform Riegl LMS-Q560 instrument (RIEGL, 2012) operating at $1550 \mathrm{~nm}$ wavelength (Höfle et al., 2008) from a light aircraft. The flying altitude was $500 \mathrm{~m}$ above the ground level, resulting in a $0.25 \mathrm{~m}$ footprint size and average point spacing of 3.2 points $/ \mathrm{m}^{2}$. Both transmitted and received waveforms were recorded and sampled with a frequency of $1 \mathrm{GHz}$ ( $1 \mathrm{~ns}$ spacing). The default full-width at half maximum (FWHM) of the transmitted pulse of this system is about $4 \mathrm{~ns}$, which was confirmed by the calculation of the mean of transmitted pulse FWHM (after optimisation) for this study area. Mean width yielded $4.01 \mathrm{~ns}$ with standard deviation of $0.028 \mathrm{~ns}$. The total number of waveforms incident on this area equalled $38,706,62.3 \%$ of which were classified as single-peak, $20.6 \%$ as two-peak, $11.5 \%$ as three-peak and $5.6 \%$ as four- or more-peak waveforms. The laser altimetry data was captured along a $75 \mathrm{~km}$-long transect line across the Yanco site. It was then extracted using the GeoCodeWF commercial software and contained (for each data record) the position of the aircraft, the train of amplitude samples of the transmitted pulse, the time offset to the beginning of recording of the returned waveform, the direction vector and the train of amplitude samples of the returned waveform.

\subsection{Aerial photography}

The aerial photography was taken using an 11 MegaPixel Canon EOS-1Ds digital camera fitted with a $34 \mathrm{~mm}$ lens, mounted on the same aircraft during the LiDAR acquisition, providing high resolution imagery over the focus area. The ground pixel size of those images is about $15 \mathrm{~cm}$. The camera was not calibrated and nor were the parameters of the camera orientation determined. However, because the study area is quite small and the change in ground elevation is only $4 \mathrm{~m}$, it was possible to rectify an aerial photo for the purpose of providing ground reference data for the classification. The rectification was carried out by measuring corresponding points in the aerial photo and shaded relief generated from the LiDAR data (with $25 \mathrm{~cm}$ pixel size) and by transforming the aerial photo to match the shaded relief. The transformation was performed using local weighted mean function and allowed for geo-coding the image. The rectified image was then used to generate the ground truth classification dataset. Three classes: ground, grass and orange trees were manually delineated to produce a land cover map of the study area (Fig. 1).

\section{Methods}

\subsection{Gaussian decomposition}

Although other procedures exist, Gaussian decomposition seems to have become a standard approach to processing of full-waveform LiDAR data. The fitting functions and optimisation algorithms differ between authors and can be adjusted according to the application. Consequently, the algorithm used in this study follows the general Gaussian decomposition procedure and consists of two stages: initial parameter estimation and optimisation. The custom procedure was developed and applied to the recorded transmitted and received waveforms, to better 


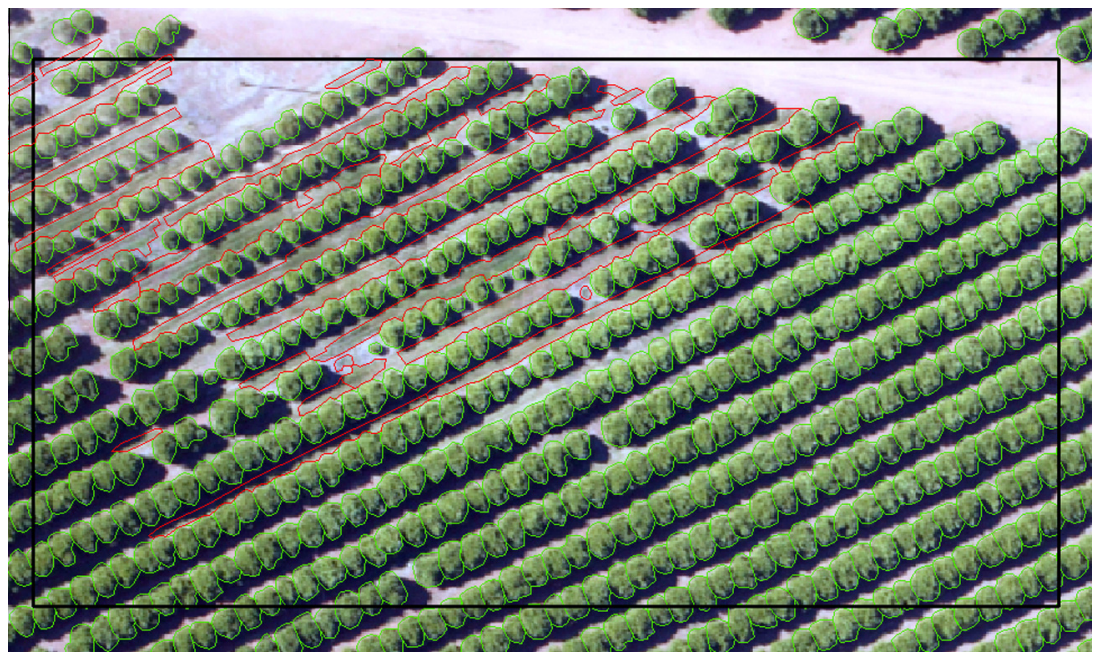

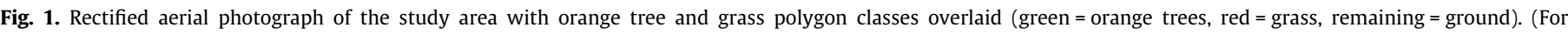
interpretation of the reference to colour in this figure legend, the reader is referred to the web version of this article.)

detect weak echoes, eliminate the ringing effect in the returns, and allow calibration of the power changes in calculating the backscattering coefficient $\gamma$.

The position, amplitude and width of the transmitted pulse are initially estimated, then optimised using a Trust Region Reflective fitting algorithm to a Gaussian function. The ringing echo amplitude ratio in the transmitted waveforms is also estimated. For received waveforms, a more sophisticated algorithm identifies overlapping pulses by detecting points of inflexion, removes ringing echoes based on the measured ringing effect in transmitted waveforms, and estimates the position, amplitude and width of up to six pulses. These parameters and a constant noise level are then optimised using a Trust Region Reflective algorithm and Gaussian function for fitting pulse shape. The optimisation procedure allows for more than six pulses to be detected. Pulse width is constrained between approximately $3 \mathrm{~ns}$ and $6 \mathrm{~ns}$, amplitude is limited to positive values and additional pulses are sought in the residuals after initial optimisation. The choice of fitting function was motivated by a study conducted by Mallet et al. (2011), who investigated the performance of several fitting functions and their relevance for classification purposes. They concluded that a decomposition method using Gaussian function is sufficient, as none of the asymmetric or flatness parameters contributed significantly to improve classification accuracy.

The performance of the above methodology is illustrated in Fig. 2 using a single orange tree as an example. Riegl RiANALYZE (Version 4.1.2, threshold parameter set to 9) decomposition detected 83 returns for this tree whereas the peak detection algorithm proposed here identified 93 peaks and optimisation raised this number to 134 points. A similar test was also carried out on a less dense Eucalyptus tree and in that case the number of points detected by RiANALYZE was nearly tripled (419 points versus 153 ). Thus, the difference in detection rate strongly depends on the density of vegetation foliage and the percentage of additionally detected peaks would be lower in urban scenes (unless densely vegetated). The Gaussian decomposition implemented in the Riegl software has been designed to serve for a wide range of sites and applications. Therefore a trade-off between the number of detected peaks and the number of ringing echoes, minimizing the latter, had to be made. The procedure proposed here is designed especially for vegetated areas and its performance in urban areas has not yet been tested.

\subsection{Data calibration}

Waveform laser scanners provide important information about the backscattering properties of the observed targets. However, to be able to explore those properties fully a radiometric calibration of the data is necessary. This is especially important if data from different campaigns, captured on different days, by different instruments and in different conditions, is to be used and compared (Höfle et al., 2008; Wagner, 2010). The calibration can be done using external artificial targets of known backscatter characteristics deployed on site before LiDAR acquisition, or using a 'natural' surface, whose reflectance is field-measured or known (Wagner, 2010). The standard approach involves extraction of backscatter cross-section and its derivatives based on the radar equation (Briese et al., 2008; Lehner and Briese, 2010; Roncat et al., 2011; Wagner, 2010; Wagner et al., 2006). Rearranging the radar equation to extract backscatter cross-section $\sigma$ allows for definition of a calibration constant:

$C_{\mathrm{cal}}=\frac{4 \pi \beta_{t}^{2}}{\eta_{\mathrm{atm}} \eta_{\text {sys }} D_{r}^{2} s_{s}}$

where $D_{r}$ is aperture diameter, $\beta_{t}$ is beam width, $s_{s}$ is full-width at half maximum of transmitted pulse expressed in terms of standard deviation, $\eta_{\text {atm }}$ is the atmospheric transmission factor, $\eta_{\text {sys }}$ is the system transmission factor. Atmospheric conditions were assumed constant throughout the acquisition area due to lack of such data, thus requiring the atmospheric transmission factor to be included in the calibration constant in Eq. (1). Furthermore, following the findings of Roncat et al. (2011), who presented an analysis of the sensitivity of the calibration constant to variations of width and the amplitude of the transmitted pulse, the amplitude of system waveform $\widehat{S}_{i}$ was kept outside the constant whereas the system waveform width $s_{s}$ was assumed to be constant (Eq. (1)).

Calibration of the LiDAR data was performed using small samples of flat asphalt road. The points with high amplitude (white markings on the road) were removed prior to processing. All samples were processed in the same way, in that both transmitted and received waveforms single peaks were detected and used as initial parameters in the optimisation of the Gaussian fit using the TrustRegion-Reflective algorithm. The optimised values of peak parameters were then used to calculate the calibration constant from the radar equation, with the road assumed to behave like an ideal 
A
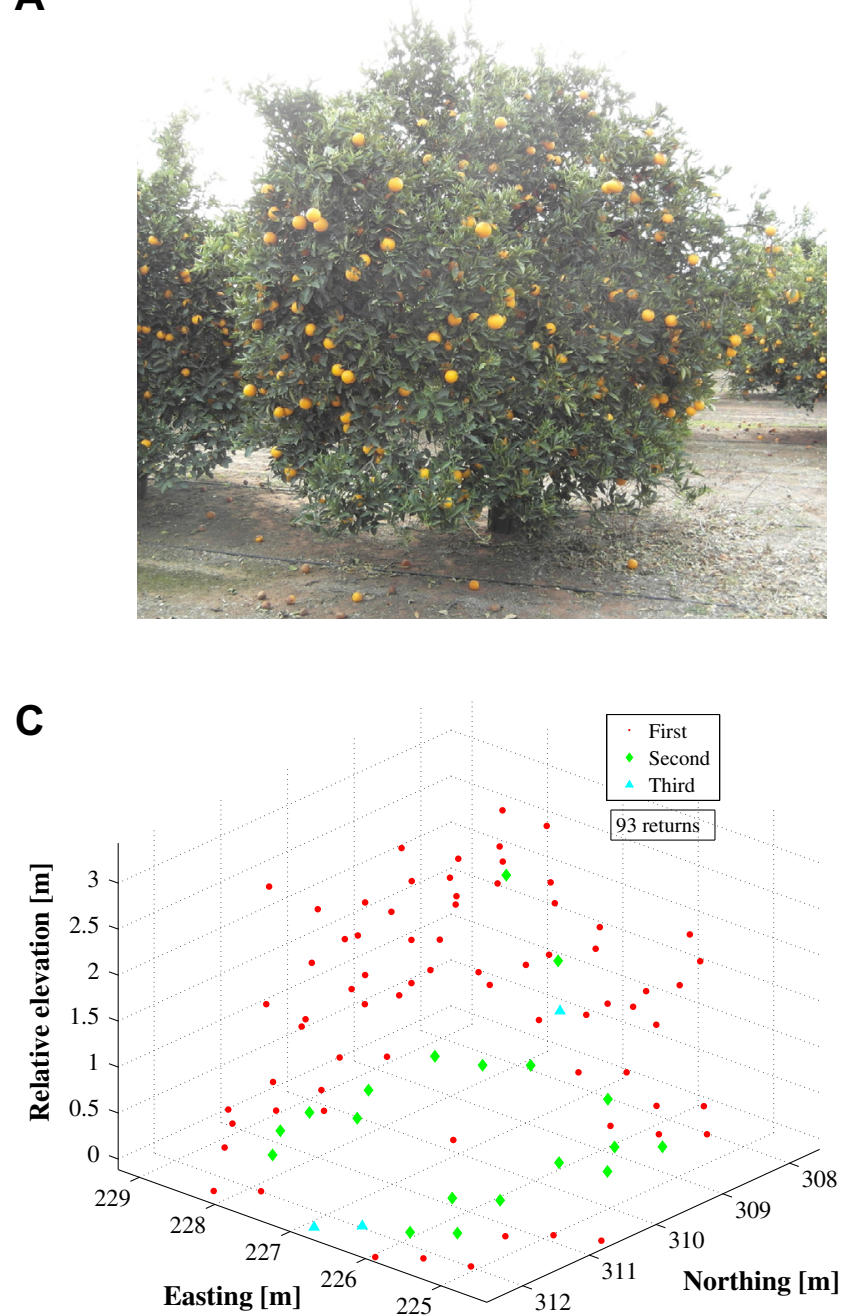

B

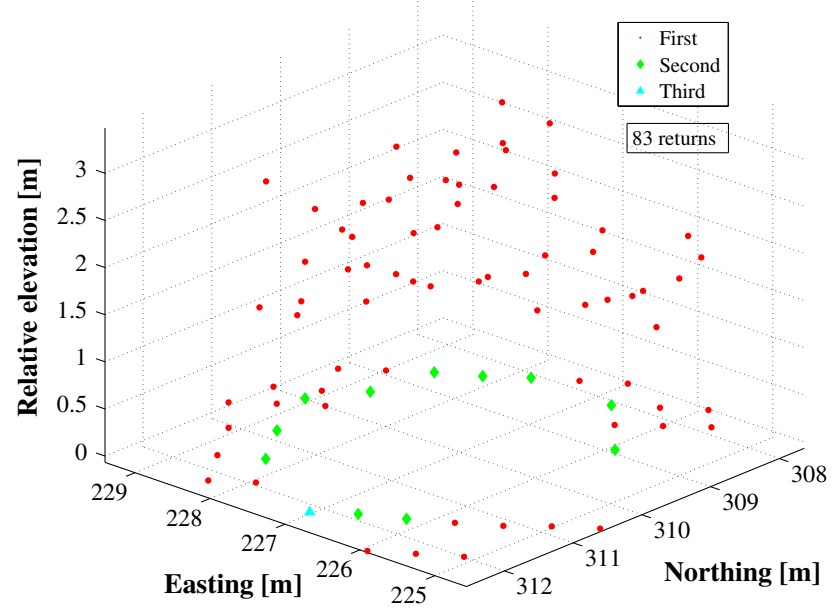

D

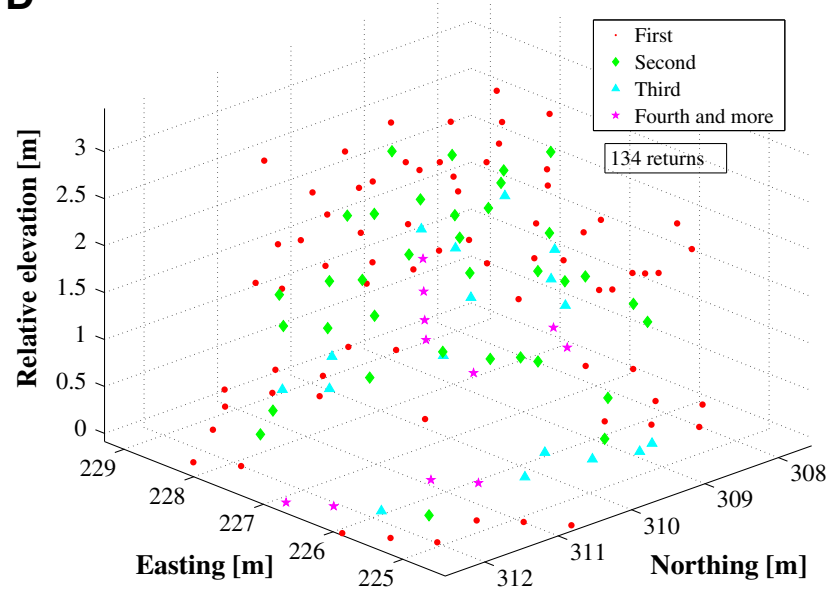

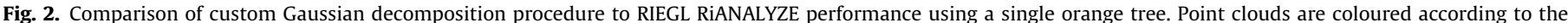

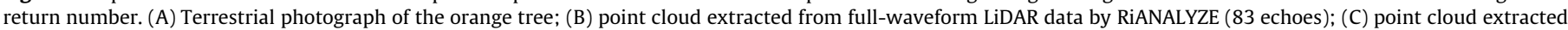

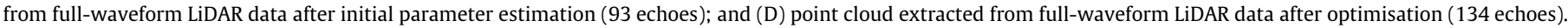
(For interpretation of the references to colour in this figure legend, the reader is referred to the web version of this article.)

Lambertian scatterer with reflectance of $\rho=0.2$ (Wagner et al., 2006). The calibration constant for each of the road points $C_{\mathrm{cal}}(i)$ was then calculated as:

$C_{\text {cal }}(i)=\frac{0.2 \pi \beta_{t}^{2} \widehat{S}_{i}}{R_{i}^{2} \widehat{P}_{i} s_{p, i}}$

where $\widehat{P}_{i}$ is the amplitude of $i$ th component in the received waveform (in the case of calibration, the only component as only single-peak waveforms were used), $R_{i}$ is range, and $s_{p, i}$ is the fullwidth at half maximum of received waveform (FWHM) expressed in terms of standard deviation. The calibration constant for the whole dataset $C_{\text {cal }}$ was calculated as the mean value of the calibration constants of all road points.

Due to the fact that backscatter cross-section is influenced by the incidence angle between the laser beam and the scattering surface, and by the effective illuminated area, Wagner (2010) recommended using backscattering coefficient $\gamma$ instead, which is free from the influence of airplane altitude. Backscattering coefficient $\gamma$ is the backscatter cross-section normalized by the laser footprint $A_{\mathrm{lf}}$ and is expressed in either $\mathrm{m}^{2} / \mathrm{m}^{2}$ or $\mathrm{dB}$.

$\gamma=\frac{\sigma}{A_{\mathrm{lf}}}=\frac{4 \sigma}{\pi R^{2} \beta_{t}^{2}}$
With the calibration constant known, it was then used to derive the backscattering coefficient $\gamma$ for each pulse within the waveform train.

$\gamma_{i}=C_{\mathrm{cal}} \frac{4 R_{i}^{2} \widehat{P}_{i} S_{p, i}}{\pi \widehat{S}_{i} \beta_{t}^{2}}$

The backscattering coefficient $\gamma$ was then employed as a calibrated radiometric parameter in the classification procedure rather than amplitude, following the recommendation of Wagner (2010) and the analysis of Alexander et al. (2010) that showed that backscattering coefficient in combination with other parameters produced the best classification accuracy.

In order to check the correct performance of calibration, a study of the variation in the backscattering coefficient with angle, based on four acquisition flights with the same RIEGL instrument, was carried out. The returns from a number of small samples $(2 \mathrm{~m}$ radius) of asphalt road per dataset with a wide range of incidence angles were used to estimate the backscattering coefficient of the road. Fig. 3 shows that there was no indication of significant angle dependence or systematic pattern in backscattering coefficient values with incidence angle. Therefore, rather than fitting a cosine of incidence angle function to model the variation of backscatter 


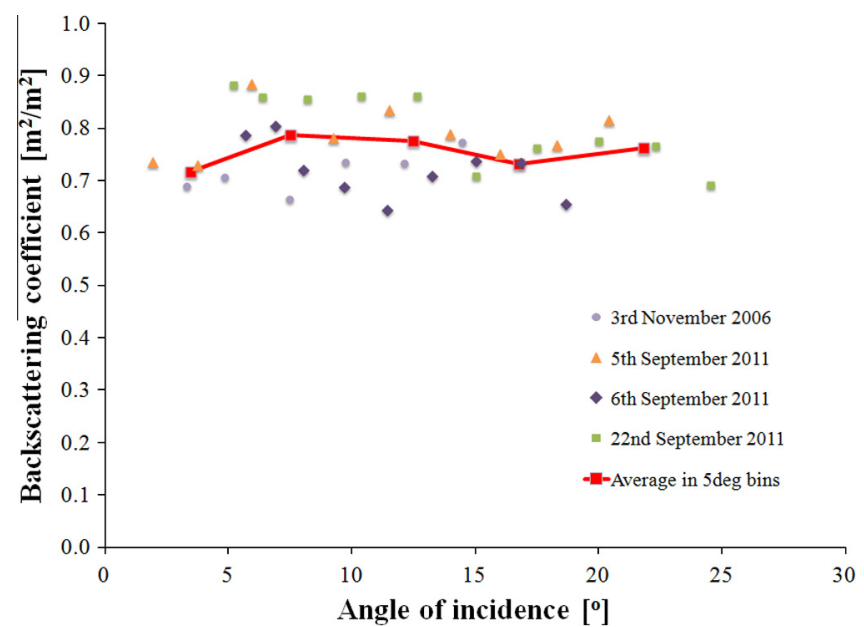

Fig. 3. Backscattering coefficient dependence on the angle of incidence using samples of asphalt road acquired on four different days with the same instrument.

for what would have been a mean adjustment of $1 \%$ over the target incidence angle range, a single calibration constant value was used.

\subsection{Reflectance}

Following Wagner (2010), backscattering coefficient relates directly to diffuse reflectance $\rho$ :

$\rho=\frac{\gamma}{4 \cos \theta}$

where $\theta$ is the incidence angle.

This equation can be used to estimate reflectance of extended targets from single-peak waveforms. However, in the case of multi-peak waveforms, derivation of reflectance is slightly more complex. As Alexander et al. (2010) noted the values of backscattering coefficient will be different for multiple and single returns as the backscatter cross-section is divided by the footprint, not by the area of each individual target. Following Wagner et al. (2008), assuming all components were triggered by the same type of target (vegetation), the backscattering coefficient of each target will be a function of the area of collision and total backscattering coefficient (sum of backscattering coefficients of all peaks within a waveform) according to:

$\gamma_{i} \approx \frac{A_{i}}{A_{\mathrm{lf}}} \sum_{i=1}^{n} \gamma_{i}$

where $A_{i}$ is illuminated area of $i$ th target and $A_{\mathrm{lf}}$ is the laser footprint. After extracting the area of collision of each target from Eq. (6), and calculating backscatter cross-section from Eq. (3) the backscattering coefficient corrected for area of collision rather than for footprint becomes:

$\gamma_{i}^{\prime}=\frac{\sigma_{i}}{A_{i}}=\frac{\gamma_{i} A_{\mathrm{lf}}}{A_{i}}=\gamma_{i} \frac{\sum_{i=1}^{n} \gamma_{i}}{\gamma_{i}}=\sum_{i=1}^{n} \gamma_{i}$

Therefore, for multiple vegetation targets the corrected version of backscattering coefficient $\gamma$ for each incidence is the total sum of backscattering coefficients within the waveform.

\subsection{DTM generation}

Ground points of single-peak waveforms were used to generate a Digital Terrain Model (DTM) for the purpose of relief removal and using relative elevation as validation for the classification. The ground points were converted into a triangular irregular network
(TIN) and then interpolated into a gridded DTM. This DTM was then subtracted from the dataset elevations. The same procedure was used to turn absolute elevation into relative elevation for each type of waveform class.

\subsection{Analysis of waveform width and backscattering coefficient}

Exploratory data analysis was carried out on the decomposed waveforms according to the number of peaks detected. Scatterplots of width versus backscattering coefficient $\gamma$, and against elevation and their histograms, were produced to define classification rules for this dataset. The derived DTM was then subtracted from the single- and multi-peak waveforms and the scatterplots of $\gamma$ and pulse width against relative elevation recreated to verify the classification performance. Although optimisation provided up to nine-peak waveforms, the analysis was only conducted on up to three-peak waveforms. This is because those three waveform groups contain about $94 \%$ of all waveforms in the dataset, and about $85 \%$ of detected points (according to the processing after optimisation). A total of 24,105 waveforms were classified as single-peak, 7990 as two-peak, 4435 as three-peak and 2176 as more than three-peak waveforms.

\subsubsection{Single-peak waveforms}

The scatterplot of $\gamma$ and pulse width of single-peak waveforms is presented in Fig. 4A. Hofton et al. (2000) assumed that the width of received waveform pulses cannot be narrower than the width of transmitted pulse, while proposing Gaussian decomposition. However, the scatterplot in Fig. 4A shows clearly that the stronger the peak the narrower its width. Moreover, the lower range of width goes down to $3.25 \mathrm{~ns}$, which is less than the width of transmitted pulse ( $4 \mathrm{~ns}$ ). This could be due to an error in the decomposition procedure. Nevertheless, since single-peak waveforms are being
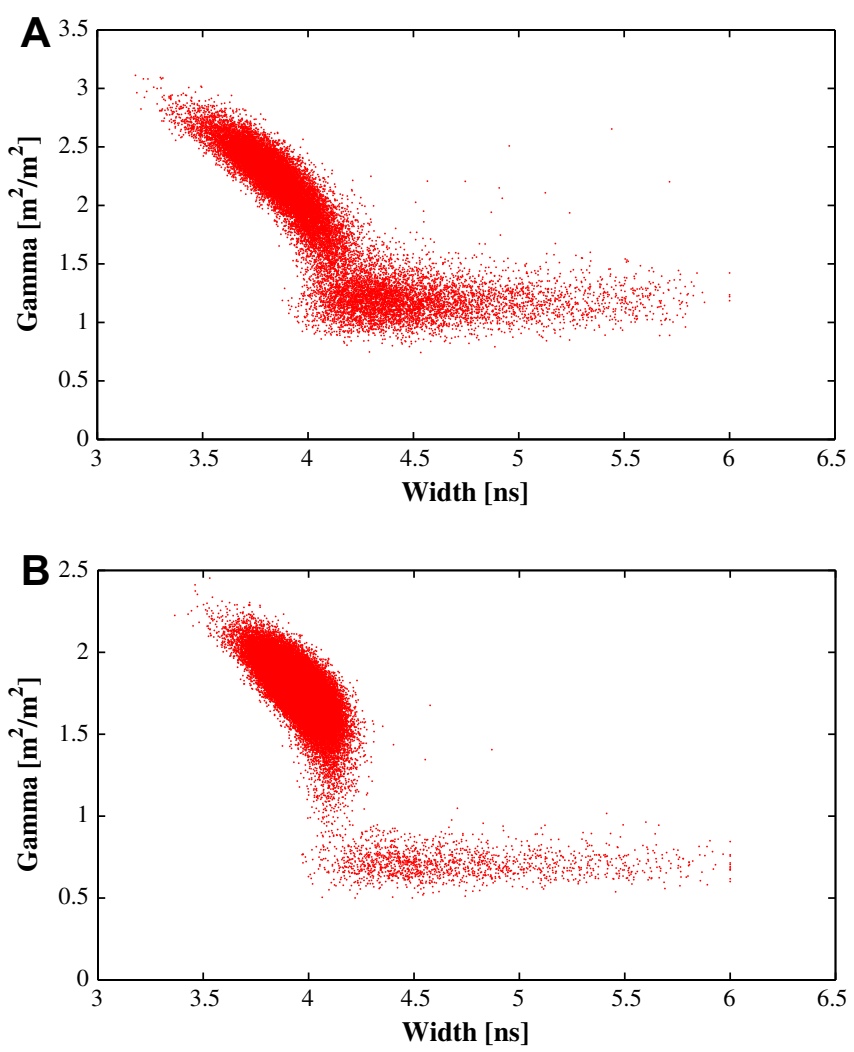

Fig. 4. Scatterplot of $\gamma$ versus pulse width of single-peak waveforms. A. Orange orchard site; B. almond orchard site for comparison. 
assessed here, only one component is fitted to the waveform shape (no merged pulses), making it very unlikely to be incorrect. Furthermore, a set of narrow single-peak waveforms was checked manually and confirmed to be decomposed correctly. An example of such a waveform is presented in Fig. 5 with transmitted waveform in plot $A$ and received waveform (of the same pulse) in plot $B$. Hence, this is the reason why decomposition procedure presented in this study allows for the width to be narrower (lower bound of width limit of around $3 \mathrm{~ns}$ ) than the width of the transmitted pulse (4 ns).

The distribution of points in the scatterplot (Fig. 4A) has a reclined 'armchair' shape, suggesting there are at least two different targets within these data. Persson et al. (2005) and Ducic et al. (2006) have previously observed that vegetation pulses are wider. Moreover, Wagner et al. (2008) have used pulse width to discriminate between canopy and terrain echoes. Gross et al. (2007) and Rutzinger et al. (2008) also reported low amplitudes of vegetation while Wagner et al. (2008) stated that the backscatter cross-section of terrain is higher than that of vegetation. All those findings suggest that the 'seat' of the 'armchair' should represent vegetation, whereas the 'back' of the 'armchair' corresponds to terrain echoes. However, the separation point is not clear. Wagner et al. (2008) used a pulse width of $4.47 \mathrm{~ns}$ (1.9 ns standard deviation) as the separation threshold of ground and vegetation returns. Examining the scatterplot of Fig. 4A, it would appear that this threshold is slightly artificial. To help decide on the separation threshold, scatterplots of pulse width and $\gamma$ coefficient against elevation, as well as histograms were produced (Fig. 6).

The scatterplots against elevation of single-peak waveforms show clear separation of ground and high vegetation (orange tree) classes, despite the four metre ground elevation variation across the site. Moreover, a tail emanating from the ground point cluster is visible in both scatterplots: low elevation points with low $\gamma$ as well as low elevation points with larger pulse width. Additionally, both the histograms of $\gamma$ and pulse width show bimodal (or even tri-modal in case of $\gamma$ ) distributions. Therefore, to find out what classes could be separated and to check whether the points representing the tail in the $\gamma$ plot (Fig. 6A) correspond to the tail in the pulse width plot (Fig. 6C), simple empirical thresholds on elevation, width and $\gamma$ were defined and classes mapped. A decision tree was constructed based on these thresholds (Fig. 7). Such a decision tree approach has been widely applied to remotely sensed data before and it has several advantages (Ducic et al., 2006). This method was chosen as its intuitive classification structure facilitates an easy definition of which parameters contribute to the discrimination between the classes. The method is also straightforward to understand, the dimension of the analysed data is low and the analysed features have physical meaning and therefore allow for determination of simple thresholds.

Classification was performed on single-peak waveforms using this approach, confirming that the 'tail' points from the $\gamma$ scatterplot in Fig. 6A correspond to the 'tail' points in the width plot in Fig. 6C, as shown in Fig. 8. Furthermore, they also represent the
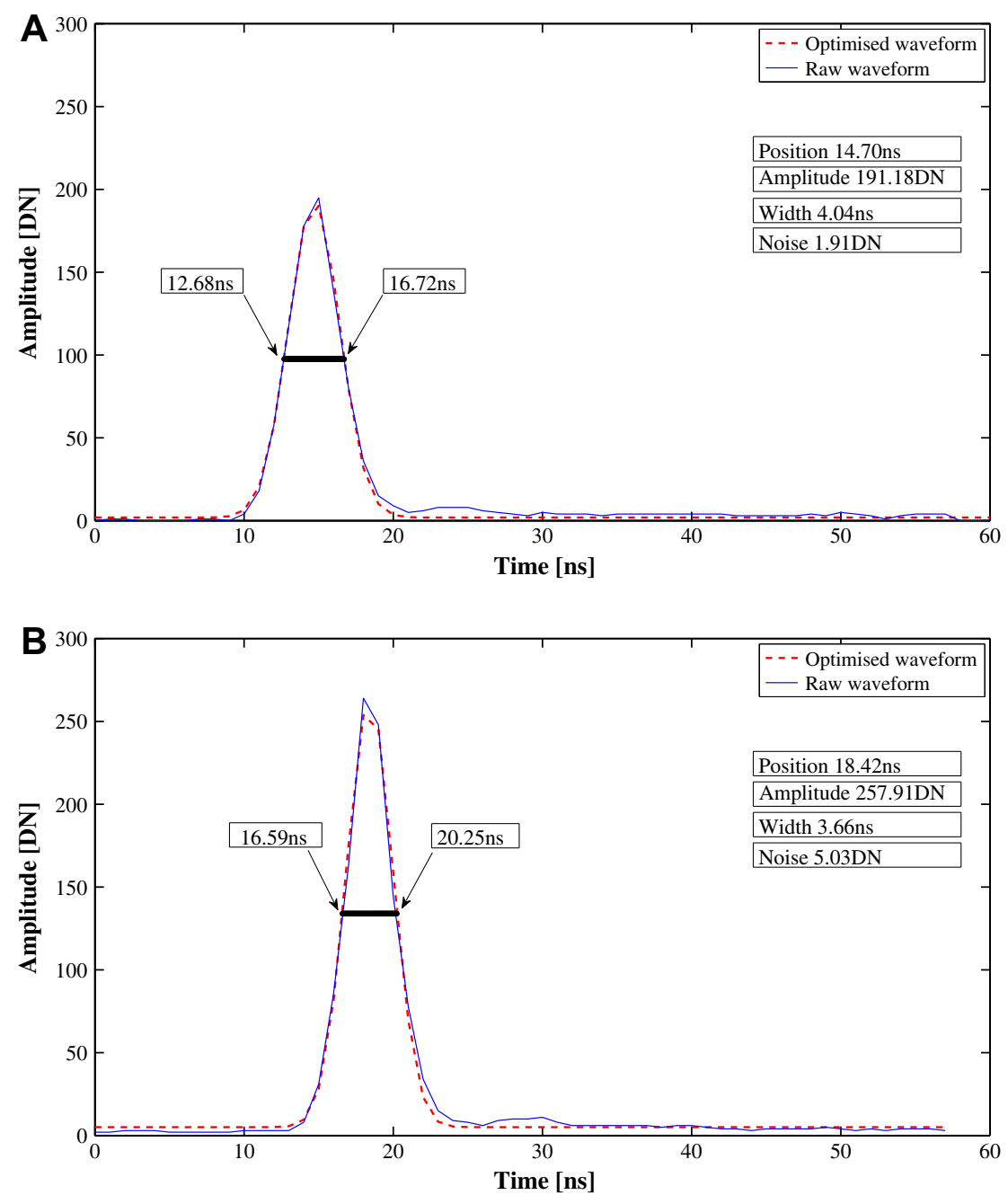

Fig. 5. An example of a transmitted waveform (A) with width of $4 \mathrm{~ns}$ and corresponding received waveform (B) with width smaller than $4 \mathrm{~ns}$. 

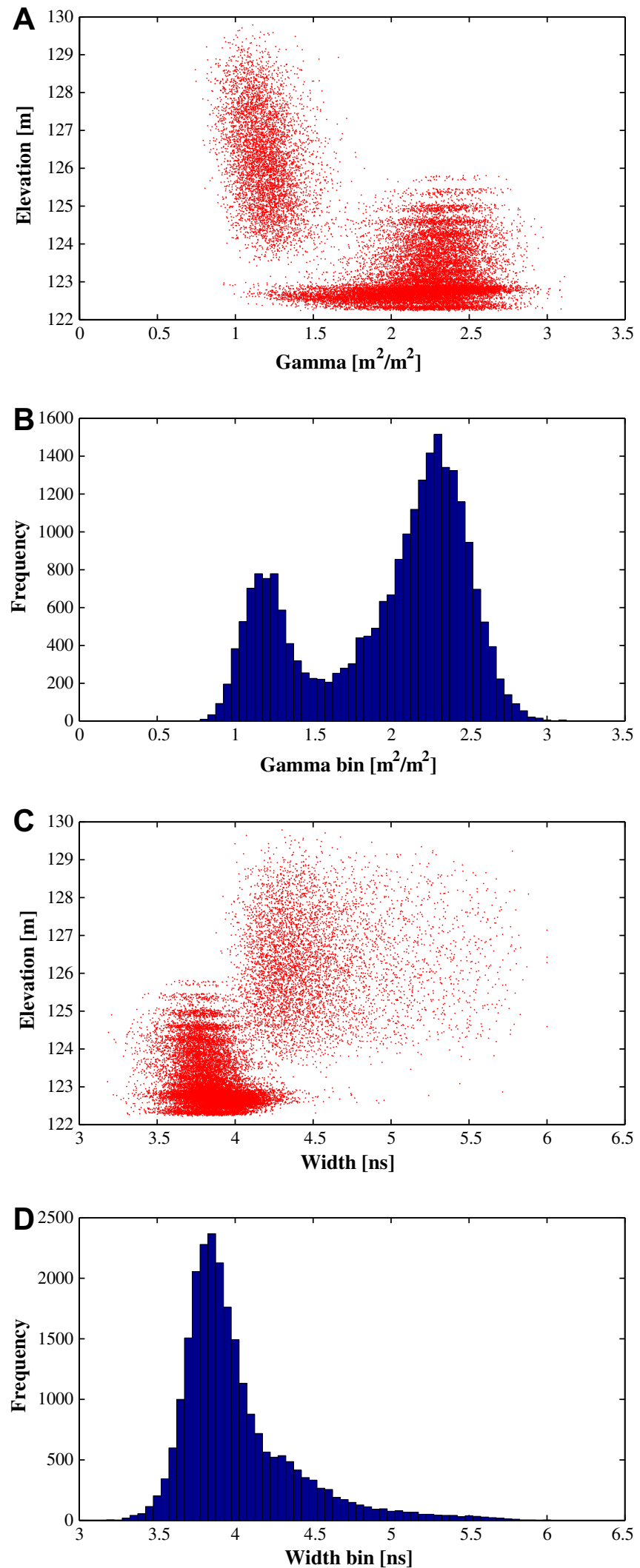

Fig. 6. Scatterplots and histograms of $\gamma$ and pulse width waveform parameters for single-peak waveforms. (A) Scatterplot of $\gamma$ versus elevation; (B) histogram of $\gamma$; (C) scatterplot of width versus elevation; and (D) histogram of width.

grass areas in the rectified aerial photography (Fig. 8B), meaning it should be possible to separate three classes from these data (ground, orange trees and grass). It also confirms the observation made by Gross et al. (2007) that intensity (or in this case $\gamma$ ) for trees is lower than for grass. The points not fulfilling the defined conditions were left unclassified, with most of them, however, seeming to correspond to grass (Fig. 8B). Fig. 8 shows that ground points were separated correctly from orange tree points. This was visually checked in the FUSION (McGaughey, 2012) visualisation software 3D environment. No high vegetation (orange tree) points were misclassified as ground. However, visual comparison of $\gamma$ against the land cover map, suggested that grass points could be better separated from the ground class. The decision tree classification using thresholds on $\gamma$, pulse width and elevation therefore works quite well, but produces quite a few unclassified returns and is dependent on geometric information.

Classification was also undertaken using solely width and $\gamma$ parameters and their scatterplot (Fig. 4A). Three classes (ground, grass, orange trees) were separated based on empirical relations between $\gamma$ and width that were defined by analysing the plot in Fig. 8A. Ground points were distinguished from other classes on the basis that $\gamma$ needs to be larger or equal to half of the pulse width, which allowed exclusion of some of the points with lower $\gamma$, seen in FUSION as grass points, from the ground class. Orange trees were separated using $\gamma$ smaller or equal to half of the pulse width minus a shift along $\gamma$ axis, to keep the same slope of the separation equation and to follow the points classified as orange in the threshold classification. Grass points were defined as falling between those two classes, which included most previously unclassified points.

In order to verify the positioning of grass returns within gamma-width scatterplot a simple test was carried out on a different study area (almond orchard), from a different dataset, acquired with the same instrument but during different field campaign. A visit to that site took place in September 2011, the same time when the LiDAR data was acquired, and proved (together with the onsite photography) that at the time of this survey, there was no grass at that site. The scatterplot of $\gamma$ versus width is provided for comparison in Fig. 4B. That scatterplot resembles the one of the orange orchard with the difference in magnitude of backscattering coefficient, which is due to different species and different soil. It also shows a clear gap between ground and almond tree returns, the analogical place where the grass returns were classified in orange orchard gamma-width scatterplot. This suggests that the grass returns were identified correctly.

Fig. 9 presents the scatterplots of single-peak waveforms classified into those three classes. Distinguished classes were visualised in FUSION to verify the performance of the classification. Furthermore, DTM was subtracted from the data values and scatterplots of $\gamma$ and width versus relative elevation generated (Fig. 9C and D). This classification produced a very well-separated ground class with consistent single-mode $\gamma$ and width histogram. Points, with grass properties, that were previously classified as ground were successfully classified as grass. None of the orange tree points were classified as ground. In the case of grass and orange tree classes the classification worked well; nevertheless there is some degree of misclassification. This is to be expected though, as both of these classes represent vegetation whose reflectance properties are very similar.

\subsubsection{Multi-peak waveforms}

In the case of multiple returns it was assumed that first returns from a two-return waveform and the first and middle out of three returns always represent vegetation for this study area. Therefore this study does not analyse them further and only last returns are classified. Wagner et al. (2008) noted that individual returns become weaker with the increasing number of returns within the waveform and that the backscatter cross-section of the last return 


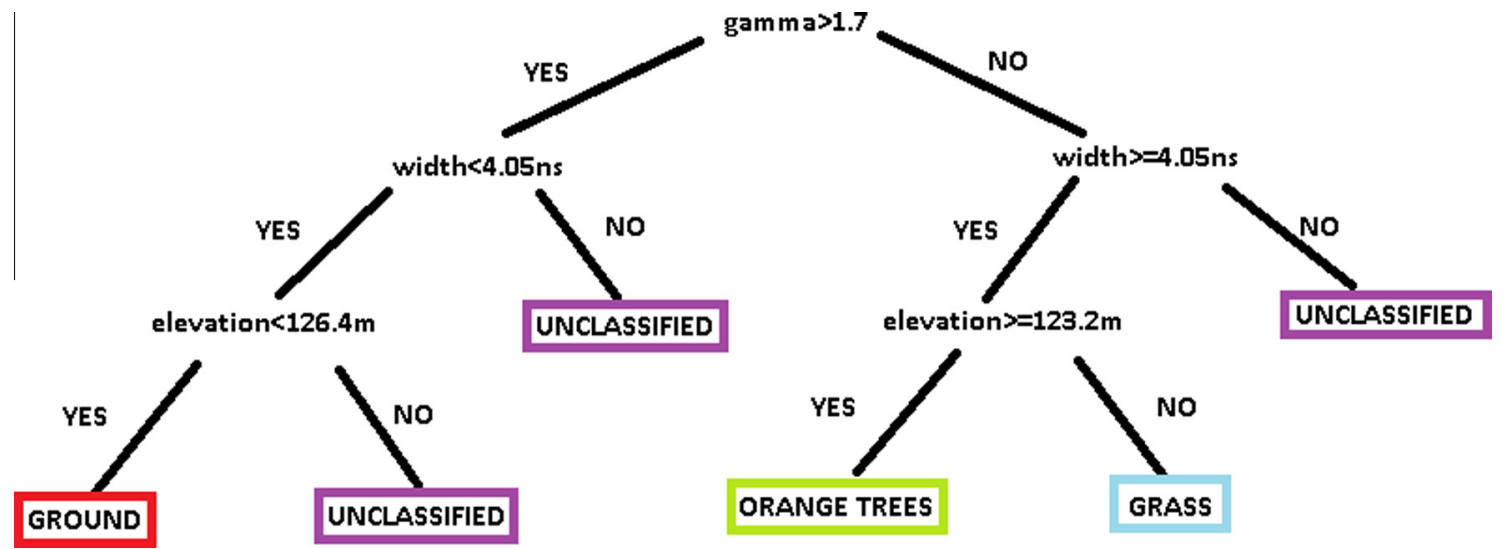

Fig. 7. Decision tree used for initial separation of four classes of single-peak waveforms.

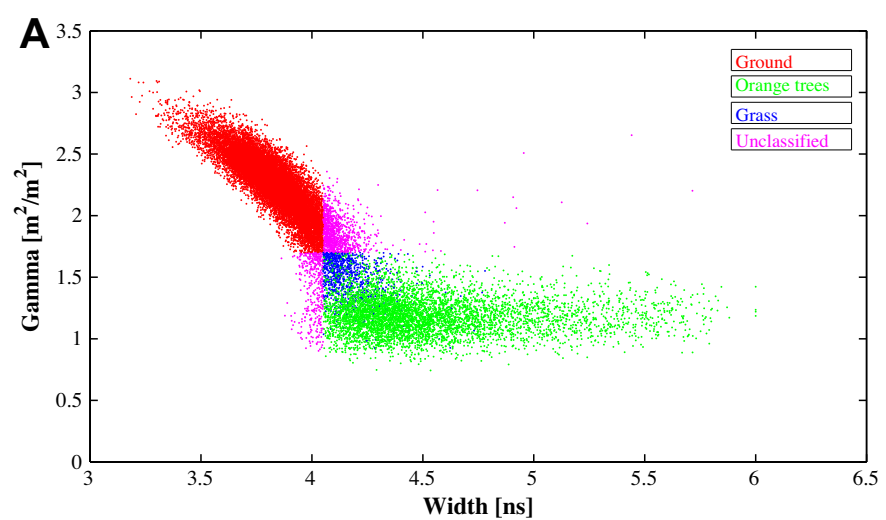

B
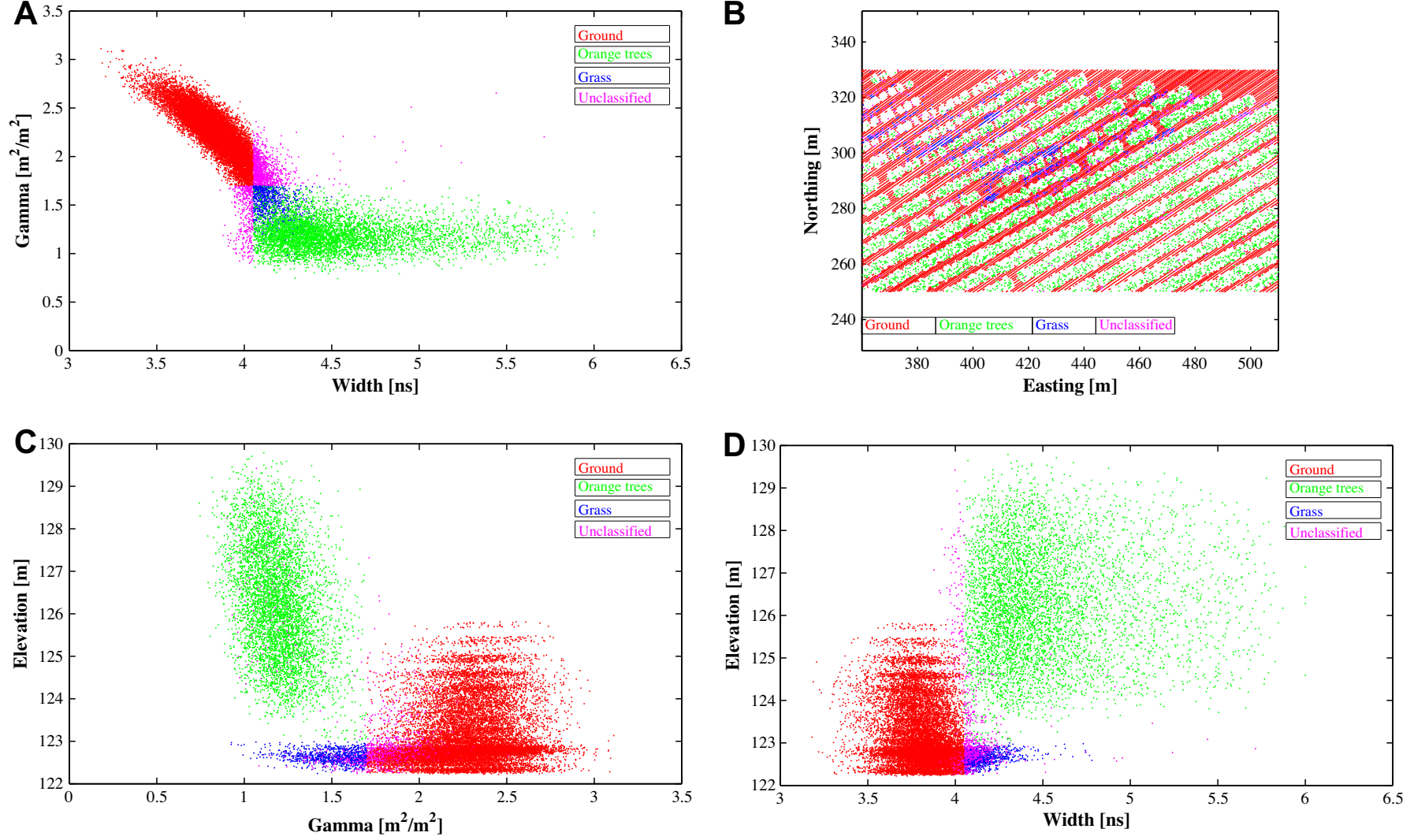

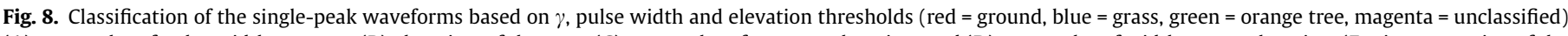

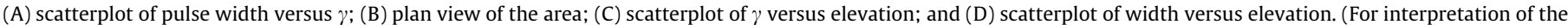
reference to colour in this figure legend, the reader is referred to the web version of this article.)

depends on the cross-section/collision area of the preceding returns. This also applies to the backscattering coefficient. The scatterplots of pulse width, elevation and $\gamma$ of last returns (Fig. 10) do not look as clear as those of single-peak waveforms. Further, their magnitude is different; they are less strong in comparison to single-peak waveforms, as predicted by Wagner et al. (2008). This is to be expected as the energy is split between more targets. Furthermore, as pointed out by Alexander et al. (2010), the values of $\gamma$ backscattering coefficient of multi-peak waveforms may be different to those of single-peak waveforms, due to the fact that backscatter cross-section is divided by the footprint area and not by the collision area of each target. For those reasons it is worth consider- ing single and multi-peak waveforms separately for the purpose of classification. Only two-peak last return distributions are presented here (Fig. 10), as the three-peak scatter-plots are very similar, just with decreasing magnitude of $\gamma$. Distribution of $\gamma$ against pulse width (Fig. 10) still has the reclined 'armchair' shape, however there is considerable amount of overlap between what should represent ground and what should represent vegetation. The interpretation of the multi-peak waveform scatter-plots is slightly more difficult.

A different approach was undertaken to separate ground returns from vegetation returns for multi-peak waveforms. For two-peak waveforms, there are two possible combinations of targets in each 


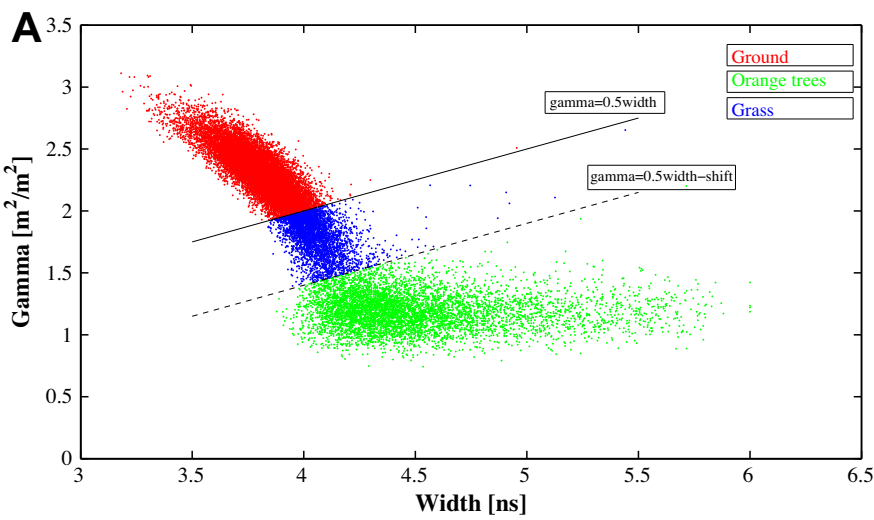

B
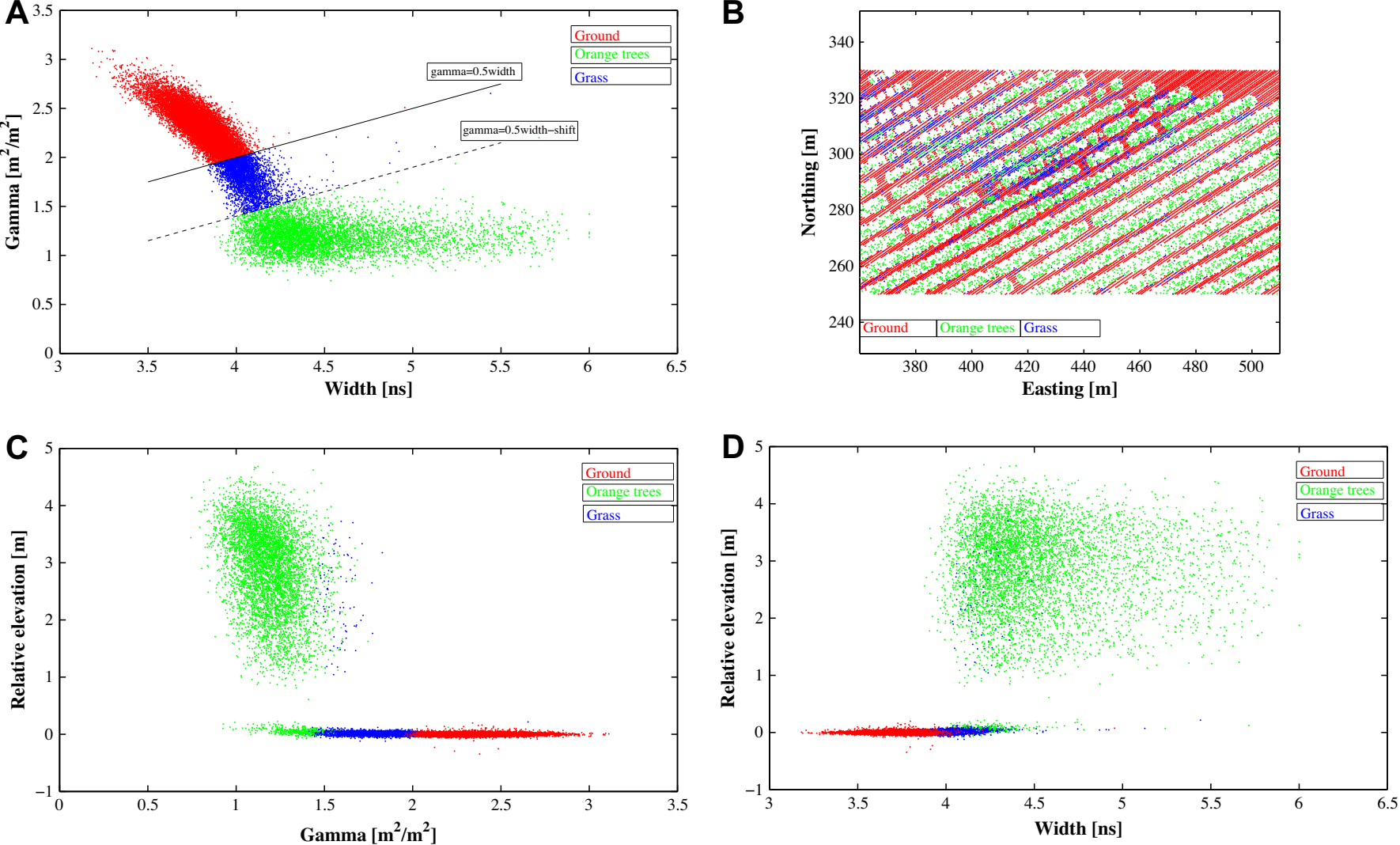

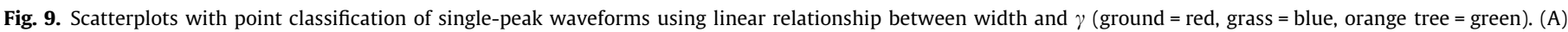

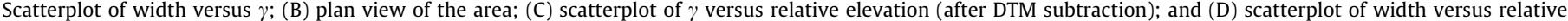
elevation (after DTM subtraction). (For interpretation of the reference to colour in this figure legend, the reader is referred to the web version of this article.)

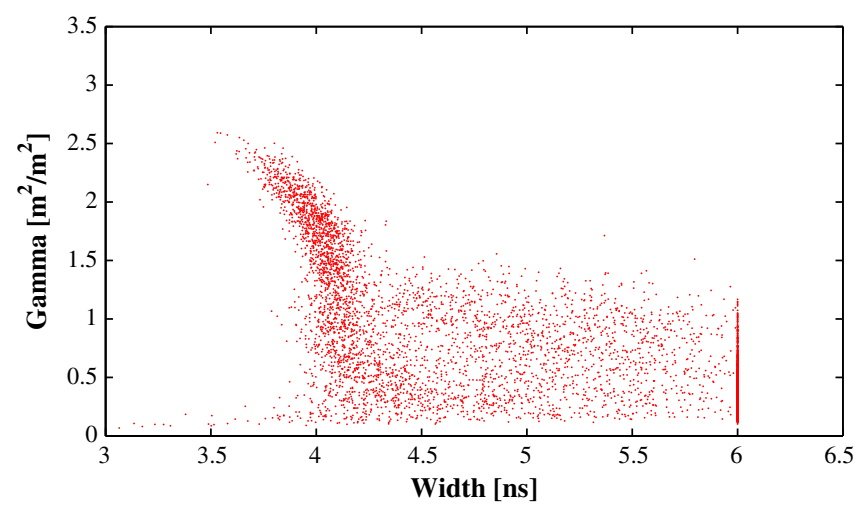

Fig. 10. Scatterplot of $\gamma$ versus width of two-peak last returns.

waveform for this study area: either both peaks originate from vegetation or the first originates from vegetation and the second from the ground. After calibration, the total light energy incident per waveform should be constant. For waveforms including multiple returns, the incident beam is divided between the two targets in proportion to the area of each intercepts, and the total returned light is a function of this and the reflectance of each of the targets. Thus, the total light returned from a set of targets with a single reflectance will be constant, however if one target has a different reflectance, the total light will vary depending on the proportions intercepted. Therefore, plotting the $\gamma$ coefficient of first return versus $\gamma$ coefficient of last returns, the distribution of vegetation-only waveform points should be at $135^{\circ}\left(45^{\circ}\right)$ to $X$ axis (Fig. 11A). Due to the higher reflec- tance of the ground than the vegetation at $1550 \mathrm{~nm}$ wavelength, the distribution of mixed-target waveforms will be at a steeper angle, as the $\gamma$ of the last return will be stronger.

Similarly, for three-peak waveforms the combination of targets would be that either all of them will represent vegetation, or the first two will represent vegetation and the last one will represent ground. Therefore, in this case the $\gamma$ of the first and middle returns were summed and plotted against the $\gamma$ of last return (Fig. 11B). The scatterplots of $\gamma$ of first (or first plus middle) against $\gamma$ of last returns were used to classify the points into two classes: ground and vegetation, by separating them using

$\gamma$ (last) $=-\gamma$ (first) + shift

Additional constraints were added to make sure that $\gamma$ of ground returns is not too low ( $>0.7)$ and width is not too large $(<4.5 \mathrm{~ns})$. The results of the two-class classification are presented in Figs. 12 and 13. The classes were visually checked in FUSION, showing that ground was successfully separated from vegetated surfaces, although there was some degree of confusion between the classes and a few off-ground points were classified as ground and vice versa. Subtraction of the DTM confirmed that one orange tree point was classified as ground (both in the case of two- and three-peak waveforms) and the vegetation class contains both orange tree returns as well as some suspected grass returns. A classification refinement was therefore necessary to remove this confusion and to distinguish the grass class from the other two.

After DTM subtraction, the easiest way of separating the orange tree class from the grass/ground class would be based on elevation. Therefore, all returns with relative elevation above $0.5 \mathrm{~m}$ were classified as orange trees, points initially classified as vegetation 

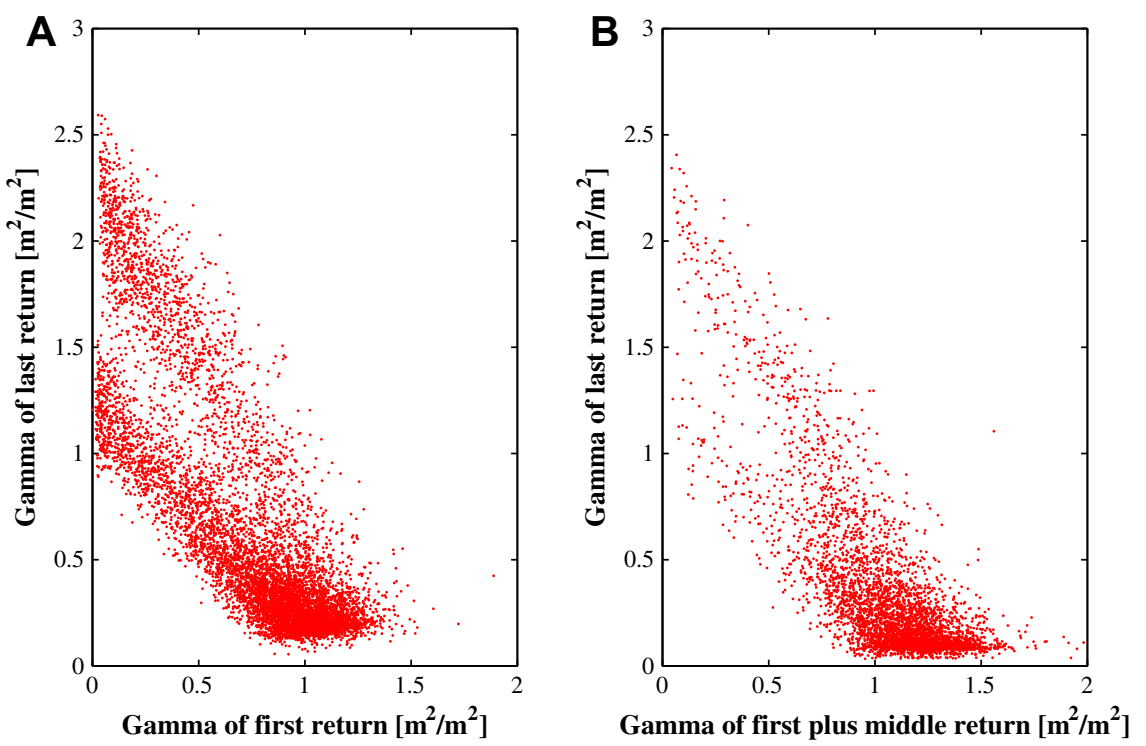

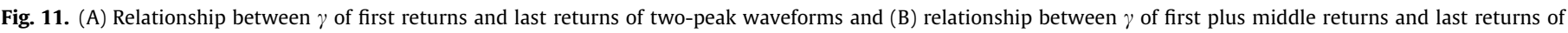
three-peak waveforms.
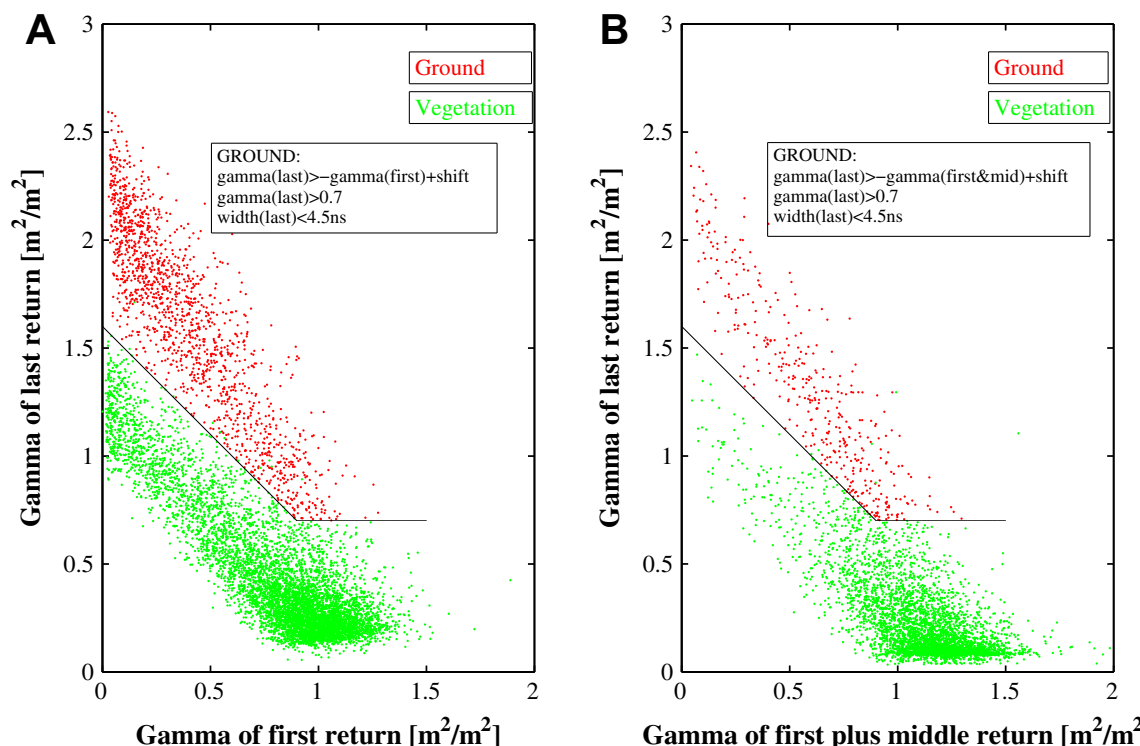

Gamma of first plus middle return $\left[\mathrm{m}^{2} / \mathrm{m}^{2}\right]$

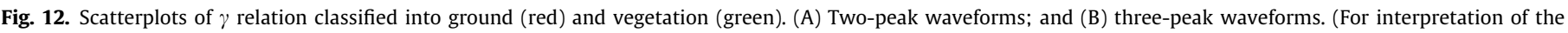
reference to colour in this figure legend, the reader is referred to the web version of this article.)

with relative elevation below $0.5 \mathrm{~m}$ were reclassified as grass, and points initially classified as ground with elevation below $0.5 \mathrm{~m}$ were unchanged. All three classes were then plotted in a $\gamma$ versus pulse width plot (Fig. 14A), which suggests that the classification could as well be carried out solely based on the gamma-width relation. The classification refinement was therefore performed again using

$\gamma(i)=(\text { width }(i)-3.2)^{-3}$

to separate grass from orange trees (from original vegetation class). To separate ground returns from grass returns, the same equation as in case single-peak waveforms was used ( $\gamma$ larger or equal to half of pulse width minus shift). The result is shown in Fig. 14B. This procedure was repeated for three-peak last returns. The initial gam- ma-gamma classification also proved to have worked well - again only one off-ground point was misclassified as ground. The grass class was then separated based on elevation threshold and finally based only on gamma-width relation using the same equations as in case of two-peak waveforms. For validation purposes, only the classification based solely on gamma-width relations was considered.

\section{Results and discussion}

\subsection{Validation using relative elevation}

The relative elevation was used to estimate accuracy in distinguishing between orange tree class and the combined classes of 

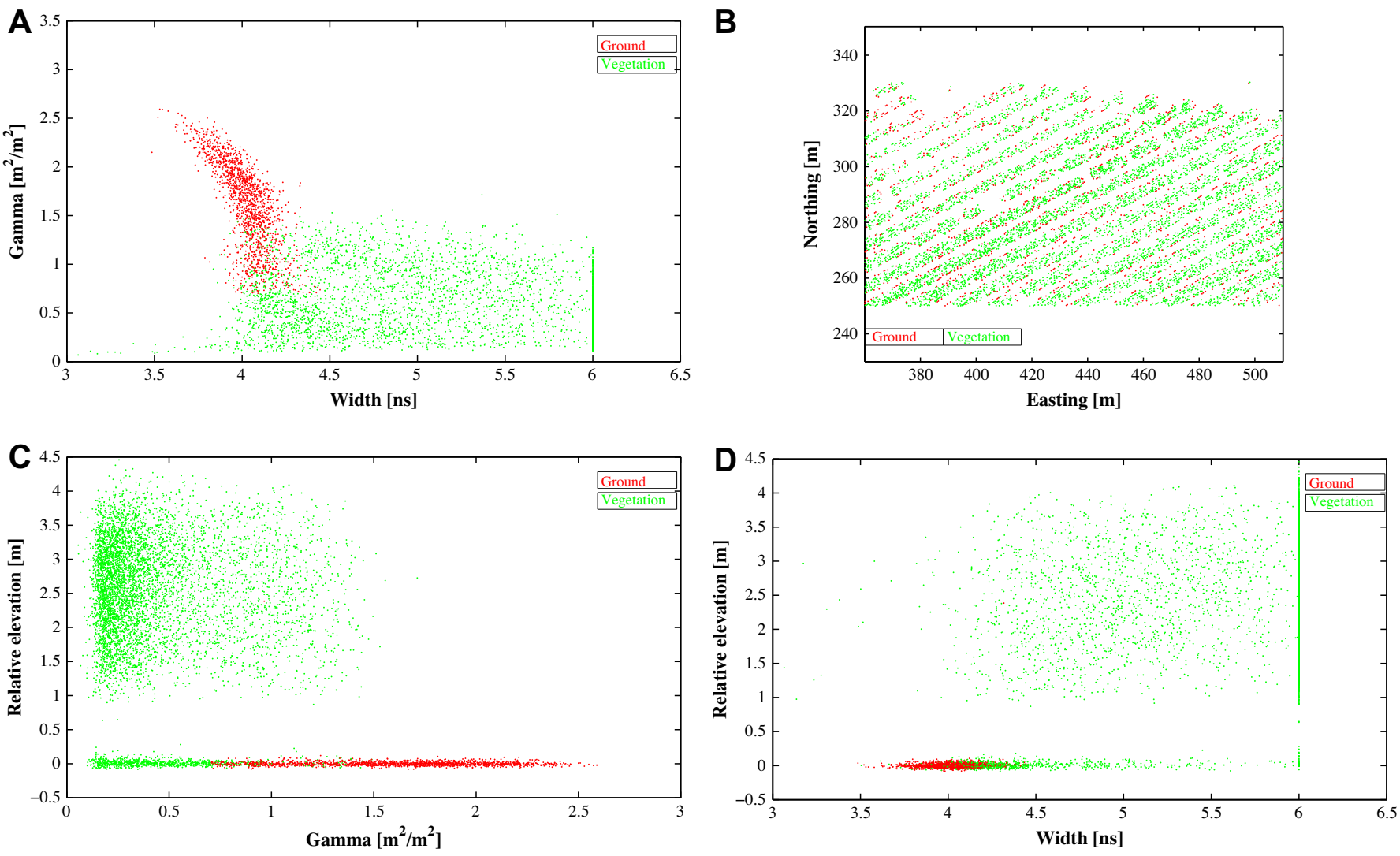

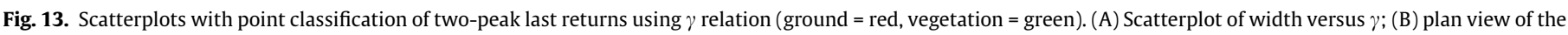

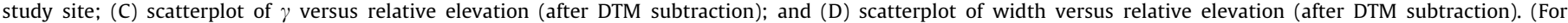
interpretation of the reference to colour in this figure legend, the reader is referred to the web version of this article.)

grass and ground in single and multi-peak datasets classified based on the gamma-width relationships alone. Ground and grass classes needed to be considered jointly for the validation as based solely on relative elevation it is difficult to verify whether the separation of those two classes have been successful, due to their similar elevation values. Therefore a threshold of $0.5 \mathrm{~m}$ above ground was used to validate whether the points were classified correctly as orange trees or grass/ground.

\subsubsection{Single-peak waveforms}

Table 1 presents a confusion matrix for the separation of orange trees from the combined grass/ground class based on validation using the $0.5 \mathrm{~m}$ elevation threshold for single-peak waveforms. The number of grass and ground classified points are also presented separately to emphasise that none of the orange tree points were misclassified as ground returns. The overall classification accuracy of the two classes reached almost 98\% with Cohen's $\kappa$ coefficient of 0.94. Cohen's $\kappa$ coefficient is a statistical measure of agreement that takes into account observed agreement and hypothetical probability of chance agreement between two classes only. Landis and Koch (1977) provided guidelines on how to interpret the $\kappa$ values. They characterized values $<0$ as indicating no agreement, $0-0.20$ as slight, $0.21-0.40$ as fair, $0.41-0.60$ as moderate, $0.61-0.80$ as substantial, and $0.81-1$ as almost perfect agreement.

\subsubsection{Multi-peak waveforms}

Classification accuracy was calculated for both two- (Table 2) and three-peak (Table 3) last returns for orange tree and combined ground/grass classes, based on relative elevation verifica- tion. The accuracy of two-peak waveform classification is not as high as the one of single-peak waveforms, nevertheless it is still very high (almost 95\% with $\kappa=0.88$ ). The accuracy of classification of three-peak waveforms is much lower though - only about $78 \%$ with $\kappa=0.56$. This may be due to the fact that the more returns within the waveform the higher the probability of merged pulses and inaccurate component fitting. Presumably it is also because of the greater partition of energy between targets. Gamma has a much larger range for any one target if it is not the only target reflecting back light. The most reflective target could only have a small amount of light fall on it and give it a small estimate of $\gamma$.

\subsubsection{Combination of all returns}

The results of the classification of single-peak waveforms and last returns of two-peak and three-peak waveforms were combined. Table 4 shows the classification accuracy achieved based on height condition for two classes. Both producer's and user's accuracies are very high, above $90 \%$ - with average accuracies of almost $95 \%$. The overall accuracy yielded nearly $95 \%$ with $\kappa$ value of 0.89 . From the DTM generation and from biomass estimation point of view, the important observation is that the classification is successful in separating ground returns - no orange tree returns were miss-classified as ground.

Fig. 15 shows histograms of elevation, $\gamma$ and pulse width for all returns. The distribution of pulse width is in most cases Gaussianlike. Pulse width tends to be the narrowest in case of ground returns and the widest for the orange tree class. The grass class has pulse width values in between the other two classes - wider than ground and narrower than orange trees. This is because those 

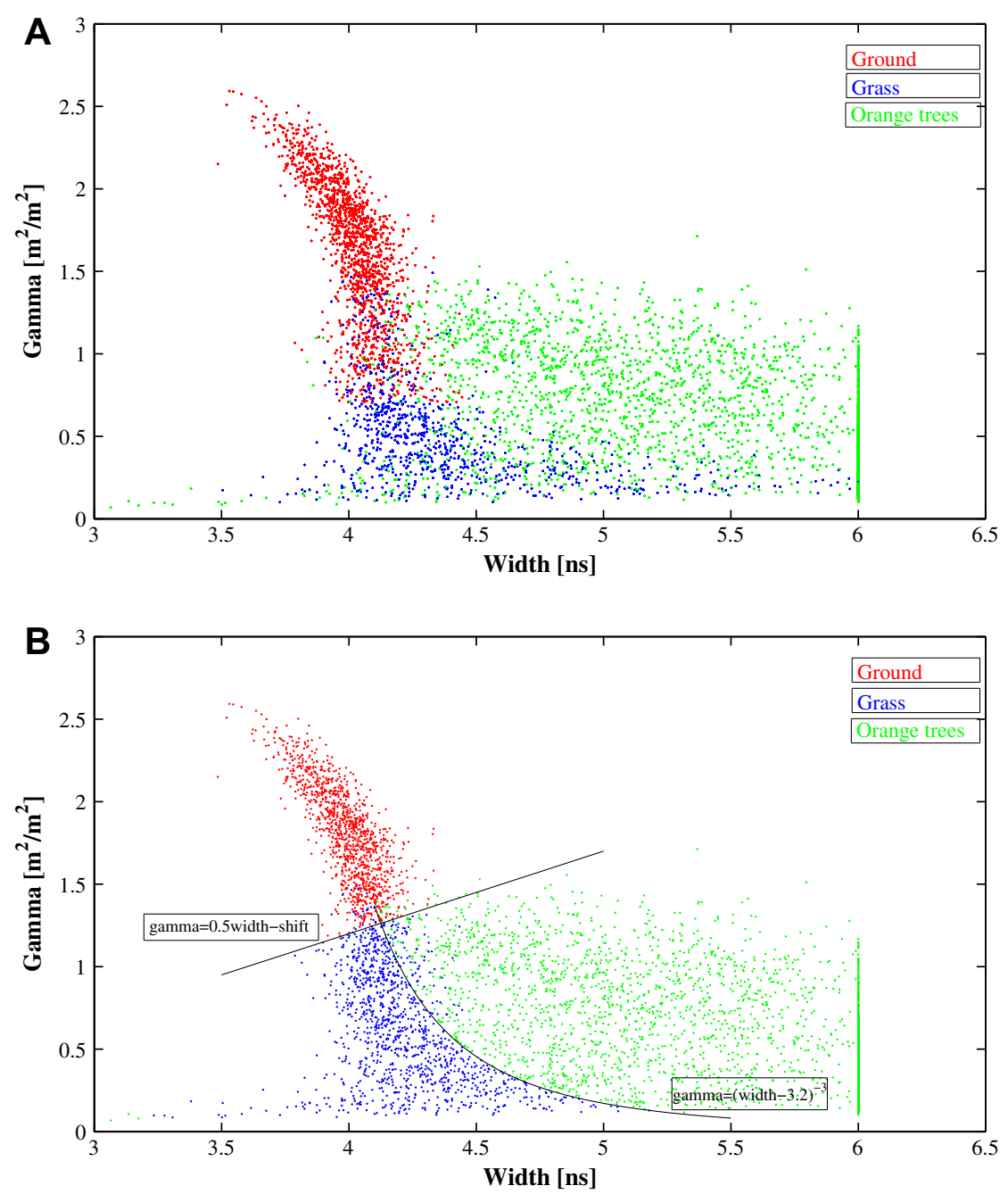

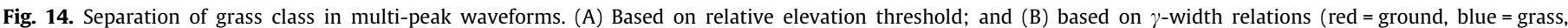
green $=$ orange tree). (For interpretation of the reference to colour in this figure legend, the reader is referred to the web version of this article.)

Table 1

Confusion matrix of gamma-width classification accuracy of single-peak waveforms assessed based on elevation.

\begin{tabular}{|c|c|c|c|c|c|}
\hline \multicolumn{6}{|c|}{$\begin{array}{l}\text { Gamma/width classification of single-peak waveforms } \\
\text { Elevation verification }\end{array}$} \\
\hline \multirow[t]{2}{*}{ Class } & \multirow[t]{2}{*}{ Orange trees } & \multicolumn{2}{|c|}{ Grass/ground } & \multirow[t]{2}{*}{ Total } & \multirow[t]{2}{*}{ Producer's accuracy } \\
\hline & & Grass & Ground & & \\
\hline \multirow[t]{2}{*}{ Orange trees } & 5534 & 83 & & 5617 & $98.5 \%$ \\
\hline & & 83 & 0 & & \\
\hline \multirow[t]{2}{*}{ Grass/ground } & 419 & 18,069 & & 18,488 & $97.7 \%$ \\
\hline & & 3451 & 14,618 & & \\
\hline Total & 5953 & 18,152 & & 24,105 & Average $98.1 \%$ \\
\hline User's accuracy & $93.0 \%$ & $99.5 \%$ & & Average 96.3\% & Overall accuracy 97.9\% \\
\hline
\end{tabular}

returns will have a mixture of properties of those two classes: grass is a vegetation type but is also close to the ground level so it is likely to have some influence from the soil (some of the light reflected from the ground and some from grass). In the case of $\gamma$, distribution is bimodal, especially for orange tree and grass classes. This is due to the fact that multi-peak $\gamma$ will differ from that of single returns. For ground returns the $\gamma$ distribution is close to Gaussian as this class has a very high percentage of single-peak waveforms in comparison to multi-peak. Furthermore, $\gamma$ values are the highest for ground and the lowest for orange tree class, as expected. For grass the $\gamma$ values are again in the middle (a mixture of properties of both classes). The thresholds used to separate different classes could be tailored depending on the application. As grass and orange tree are vegetation classes, they have similar radiometric properties and thus it is a matter of a trade-off while separating them from each other. 
Table 2

Confusion matrix of gamma-width classification accuracy of two-peak last returns assessed based on elevation.

\begin{tabular}{|c|c|c|c|c|c|}
\hline \multicolumn{6}{|c|}{$\begin{array}{l}\text { Gamma/width classification of two-peak waveforms } \\
\text { Elevation verification }\end{array}$} \\
\hline \multirow[t]{2}{*}{ Class } & \multirow[t]{2}{*}{ Orange trees } & \multicolumn{2}{|c|}{ Grass/ground } & \multirow[t]{2}{*}{ Total } & \multirow[t]{2}{*}{ Producer's accuracy } \\
\hline & & Grass & Ground & & \\
\hline \multirow[t]{2}{*}{ Orange trees } & 5427 & 187 & & 5614 & $96.7 \%$ \\
\hline & & 187 & 0 & & \\
\hline \multirow[t]{2}{*}{ Grass/ground } & 223 & 2153 & & 2376 & $90.6 \%$ \\
\hline & & 978 & 1175 & & \\
\hline Total & 5650 & 2340 & & 7990 & Average $93.7 \%$ \\
\hline User's accuracy & $96.1 \%$ & $92.0 \%$ & & Average $94.1 \%$ & Overall accuracy $94.9 \%$ \\
\hline
\end{tabular}

Table 3

Confusion matrix of gamma-width classification accuracy of three-peak last returns assessed based on elevation.

\begin{tabular}{|c|c|c|c|c|c|}
\hline \multicolumn{6}{|c|}{$\begin{array}{l}\text { Gamma/width classification of three-peak waveforms } \\
\text { Elevation verification }\end{array}$} \\
\hline \multirow[t]{2}{*}{ Class } & \multirow[t]{2}{*}{ Orange trees } & \multicolumn{2}{|c|}{ Grass/ground } & \multirow[t]{2}{*}{ Total } & \multirow[t]{2}{*}{ Producer's accuracy } \\
\hline & & Grass & Ground & & \\
\hline \multirow[t]{2}{*}{ Orange trees } & 1890 & 316 & & 2206 & $85.7 \%$ \\
\hline & & 316 & 0 & & \\
\hline \multirow[t]{2}{*}{ Grass/ground } & 659 & 1527 & & 2186 & $69.9 \%$ \\
\hline & & 1257 & 270 & & \\
\hline Total & 2549 & 1843 & & 4392 & Average $77.8 \%$ \\
\hline User's accuracy & $74.1 \%$ & $82.9 \%$ & & Average $78.5 \%$ & Overall accuracy $77.8 \%$ \\
\hline
\end{tabular}

Table 4

Confusion matrix of gamma-width classification accuracy of all investigated waveforms assessed based on elevation.

\begin{tabular}{|c|c|c|c|c|c|}
\hline \multicolumn{6}{|c|}{$\begin{array}{l}\text { Gamma/width classification of all waveforms (single and last) } \\
\text { Elevation verification }\end{array}$} \\
\hline \multirow[t]{2}{*}{ Class } & \multirow[t]{2}{*}{ Orange trees } & \multicolumn{2}{|c|}{ Grass/ground } & \multirow[t]{2}{*}{ Total } & \multirow[t]{2}{*}{ Producer's accuracy } \\
\hline & & Grass & Ground & & \\
\hline \multirow[t]{2}{*}{ Orange trees } & 12,851 & 586 & & 13,437 & $95.6 \%$ \\
\hline & & 586 & 0 & & \\
\hline \multirow[t]{2}{*}{ Grass/ground } & 1301 & 21,749 & & 23,050 & $94.4 \%$ \\
\hline & & 5686 & 16,063 & & \\
\hline Total & 14,152 & 22,335 & & 36,487 & Average $95.0 \%$ \\
\hline User's accuracy & $90.8 \%$ & $97.4 \%$ & & Average $94.1 \%$ & Overall accuracy $94.8 \%$ \\
\hline
\end{tabular}

\subsection{Validation using a combination of aerial photography and relative elevation}

Rectified aerial photography was used to verify whether the grass and ground classes were separated correctly, by counting the number of points of each class falling within the relevant land cover polygons. However, using aerial photography alone proved to be inadequate for 3D data. First, aerial photographs provide only $2 \mathrm{D}$ verification, so it is not possible to judge whether grass-classified points falling underneath the trees were classified correctly or not. A site visit, although carried out in 2011, suggests there might have been some grass and dead leaves underneath the trees when the LiDAR data were acquired. Additionally, even though ground points falling within orange tree polygons have an elevation close to the ground level, they are treated as misclassified, which is clearly incorrect. Second, the aerial photography has deep shadows which make it impossible to see whether there is grass in between the tree rows, especially in the area of denser trees. A significant amount of ground class points treated as incorrect (as orange trees) were also on the shadow side of the trees. Third, the resolution of the photography is not high enough, taking into account that grass was found to be patchy at scales less than the image resolution during the site visit in 2011. Therefore, ground classified points falling within grass polygons may actually be correctly classified. Consequently, LiDAR classification is most likely to be more accurate than a land cover maps generated from an aerial photography under these conditions.

All the above reasons led to a combination of elevation threshold and aerial photography being used to verify the accuracy of the three classes. Thus, the orange tree class was verified based on elevation threshold alone because the photography did not provide any more useful information over the elevation data. The grass class was verified based on the simplified grass polygon (that outlines the interpreted grass area) that allows grass returns that appear underneath the orange trees, and covers the North West part of the area, where the grass was interpreted to occur. The confusion matrix for classification of the three classes is presented in Table 5. Ground returns were assumed to have been classified 

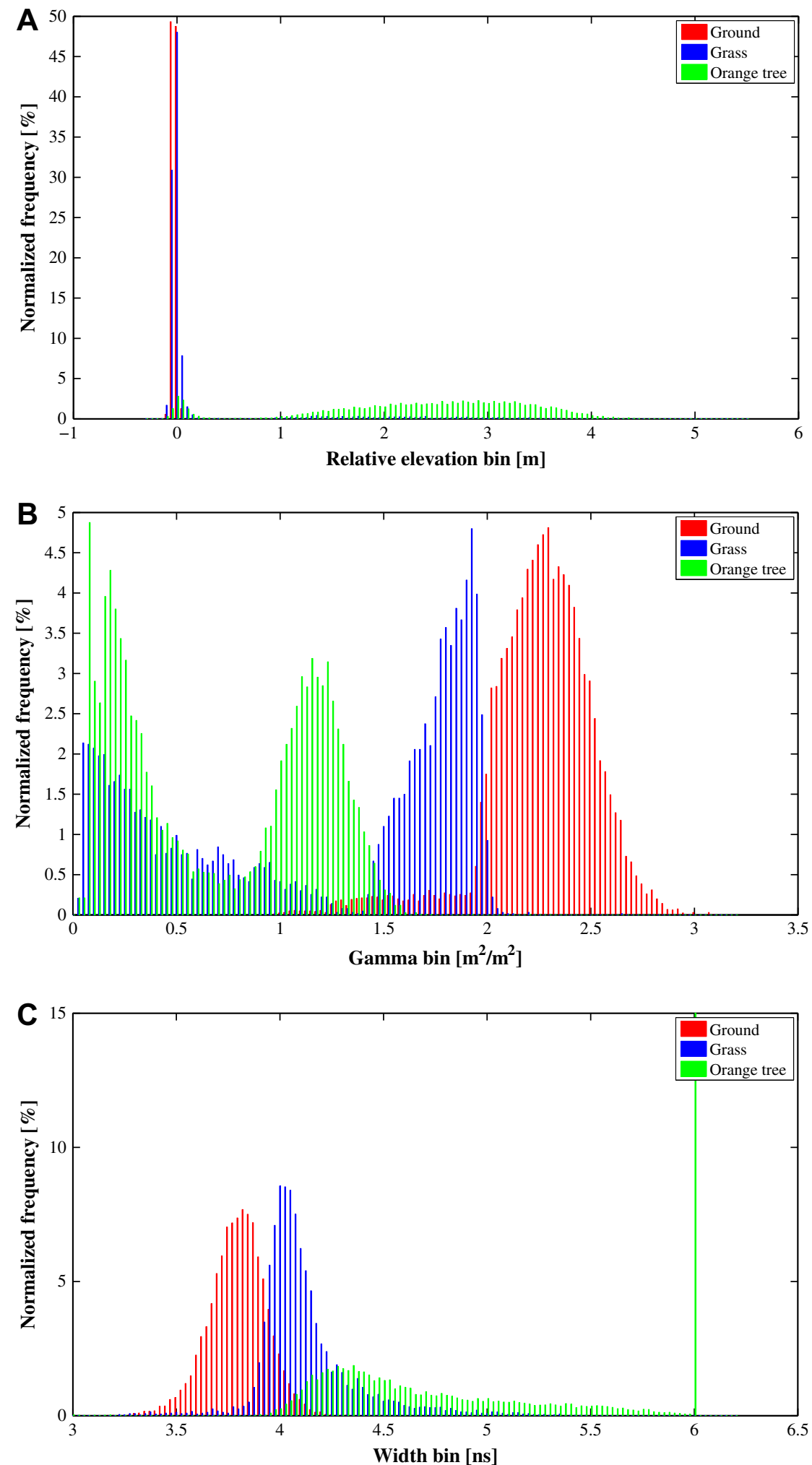

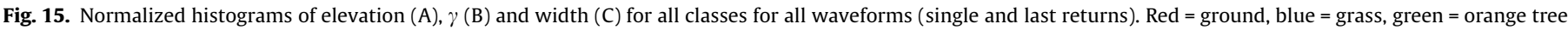
(For interpretation of the reference to colour in this figure legend, the reader is referred to the web version of this article.)

correctly - their mean relative elevation value is 0 with standard deviation of $2 \mathrm{~cm}$ and maximum value not exceeding $8 \mathrm{~cm}$. Because of that assumption, ground user's and producer's accuracies are overestimated, as is the producer's accuracy of grass, and the overall accuracy. The grass class still has the lowest accuracy how- ever this is due to a lack of precise ground truth for that class. The overall classification yielded $91 \%$ accuracy. Fig. 16 shows the final classification of single and last returns of the study area. The study area is colour-coded according to the class (Fig. 16A and B), according to the backscattering coefficient (Fig. 16C) and width (Fig. 16D). 
Table 5

Confusion matrix of gamma-width classification accuracy of all investigated waveforms assessed based on combination of elevation and aerial photography.

\begin{tabular}{|c|c|c|c|c|c|}
\hline \multicolumn{6}{|c|}{$\begin{array}{l}\text { Gamma/width classification of all waveforms (single and last) } \\
\text { Photo and elevation verification }\end{array}$} \\
\hline Class & Orange trees & Grass & Ground & Total & Producer's accuracy \\
\hline Orange trees & 12,851 & 586 & 0 & 13,437 & $95.6 \%$ \\
\hline Grass & 922 & 4337 & 0 & 5259 & $82.5 \%$ \\
\hline Ground & 379 & 1349 & 16,063 & 17,791 & $90.3 \%$ \\
\hline Total & 14,152 & 6272 & 16,063 & 36,487 & Average $89.5 \%$ \\
\hline User's accuracy & $90.8 \%$ & $69.1 \%$ & $100 \%$ & Average $86.6 \%$ & Overall accuracy $91.1 \%$ \\
\hline
\end{tabular}

\subsection{Reflectance calculation}

The Riegl laser scanner LMS-Q560 operates at $1550 \mathrm{~nm}$ wavelength. Reflectance $\rho$ at this wavelength was estimated from LiDAR returns based on Eq. (5) for ground, grass and orange tree class. The incidence angle was used disregarding the ground relief, as the site is relatively flat. As it is also very difficult to define incidence angle for vegetation, the incidence angle was defined from the direction vector, generating an effective reflectance based on the assumption that the vegetation is horizontal and continuous. For the reflectance estimation, only single-peak waveforms were used, as only those are known to hit extended targets that are the same size as or larger than the footprint size. Table 6 shows the summary of mean reflectance for all three classes. The reflectance of the ground was estimated as 0.60 , reflectance of orange trees as 0.31 , and reflectance of grass as 0.46. According to Bowker et al. (1985), the reflectance of orange leaves at $1550 \mathrm{~nm}$ is 0.32 . The soil in the study area is a mixture of sand, silt and clay. Those soil types have the following reflectance (Bowker et al., 1985): dry red clay -0.52 , dry silt -0.64 and dry sandy soil -0.44 . Taking into account that the survey was carried out after a long period of drought in the area, those values could be even higher. Therefore the reflectance of ground (0.60) and reflectance of orange trees (0.31) match the Bowker et al. (1985) study well. The ground is therefore twice as reflective as vegetation at this wavelength. The grass class, having a mixture of vegetation and ground reflectance contributions, has a reflectance value in between ground and orange trees $(0.46)$, as expected.

In the case of multi-peak waveforms, as mentioned in Sections 3.3 and 3.5.2 the values of backscattering coefficient $\gamma$ will be different to those of single-peak waveforms, due to the fact that backscatter cross-section is divided by the footprint area and not by the collision area of each target. The reflectance of those targets can nevertheless be estimated using the sum of $\gamma$ values in a waveform (Eq. (7)) on the condition that all targets within this waveform are the same. Having estimated the ground reflectance and knowing it is twice as reflective as vegetation, it is possible to estimate reflectance of all targets taking into account the relative difference between the reflectance of ground and vegetation. This was carried out for two-peak and three-peak waveforms (Table 7). Apart from the reflectance of the orange tree class calculated from three-peak waveforms, all reflectance values are consistent. In most cases reflectance derived from three-peak waveforms has relatively high standard deviation. This is due to the fact that it is more difficult to estimate the Gaussian parameters when there are more components in the waveform.

\subsection{Further discussion}

A couple of curiosities have been identified while carrying out work on this study site. The first one is that the received pulse width in some cases tends to be smaller than that of transmitted pulse. This is not necessarily peculiar to this dataset as the width is often constrained to be equal or greater than that of transmitted pulse in width estimation techniques (Hofton et al., 2000). As discussed in Section 3.5.1 narrow widths could be a result of an error in decomposition procedure. For example if the noise level was overestimated this would cause the measurement of width being performed further up the peak where the width of the pulse decreases; or if two peaks were fitted into one causing their widths to be much narrower. As for the overestimation of noise level, the authors have some confidence that this was not the case as the estimation of initial parameters for input into trust-regionreflective algorithm disregarded the noise level at all and still provided width estimates narrower than that of emitted pulse. Fitting of two pulses into one peak as a cause of narrow width was also ruled out by manually investigating a subset of single-peak waveforms (with only one component fitted) with narrow pulse widths. These turned out to be correctly decomposed; the pulses appeared normal, they were just somewhat narrower than expected. Possible ways in which only part of the pulse could be returned by a reflecting component have been considered, for instance it is possible that as the beam scans, the leading edge of the outgoing pulse may be reflected whereas the trailing edge misses the reflector, such that only a truncated return is recorded. However, the estimate of the horizontal within-pulse displacement would be at around $0.03 \mathrm{~mm}$ in the data used in this study. Thus any pulse that would have had missed a reflector at its end would only have had a grazing reflection at the pulse start, far too slight for a measurable return. It is hard to see how the geometry of the vegetation or ground could otherwise narrow the pulse so much, thus, the only remaining and more plausible reason behind it could be some shortcoming in the return light digitisation hardware or software that does not affect the outgoing pulse digitisation.

The second peculiarity identified is related to the lack of dependence of the backscattering coefficient of the asphalt road samples on the angle of incidence. As presented in Section 3.2 a set of small samples of points reflected from a road surface with varying incidence angle of four different acquisitions were investigated. They showed that any angular dependence is actually much smaller than other factors such as surface variability. This is similar to findings of Lehner and Briese (2010) who also showed that the noise is much greater than the trend in angular dependence of backscattering coefficient. Lehner and Briese (2010) did show a slight decrease in backscattering coefficient for angles above approximately 17 , but still far lower than the exhibited scatter.

\section{Conclusions and future work}

This study has examined the suitability of waveform parameters such as backscattering coefficient $\gamma$ and pulse width for rural scene classification, without the need to use geometric or neighbourhood relations. The approach first classifies waveforms according to the number of components, and then treats those groups separately. Due to normalization of backscatter cross-section by the laser footprint, the backscattering coefficient values 
A

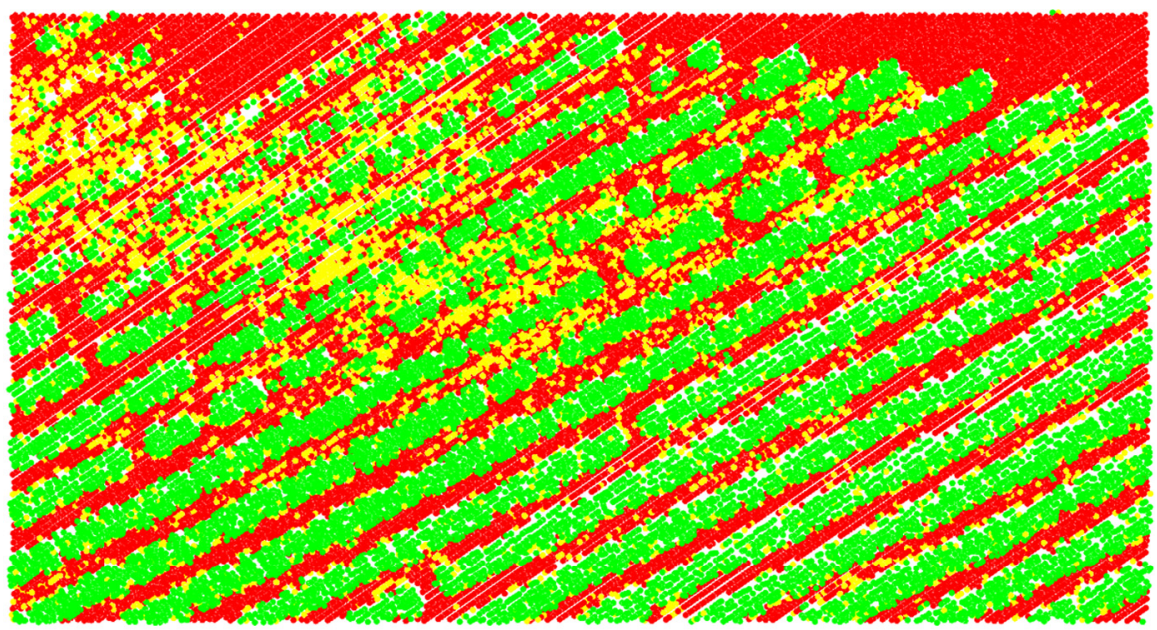

B
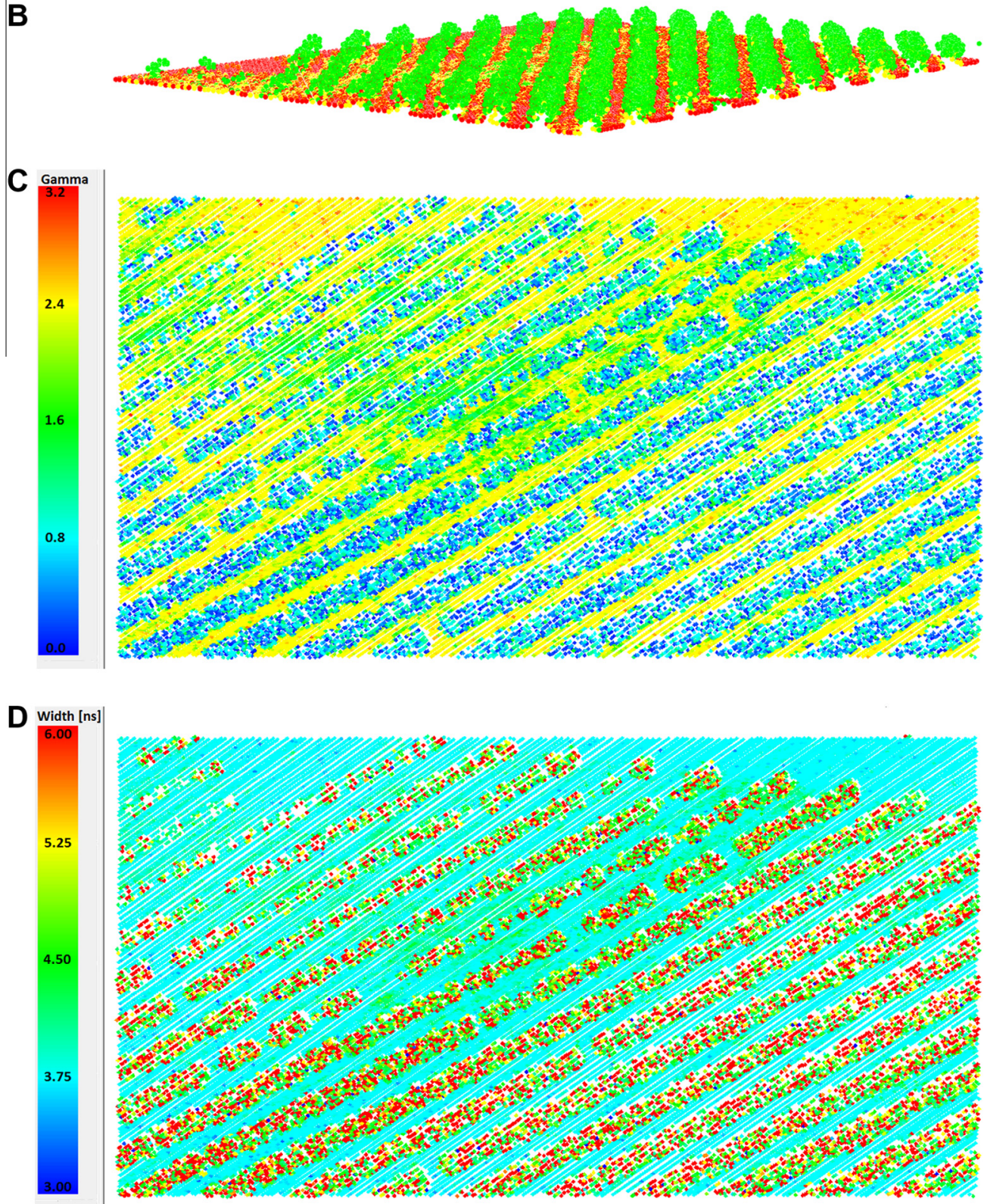

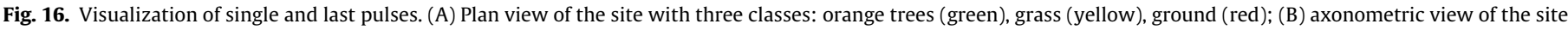

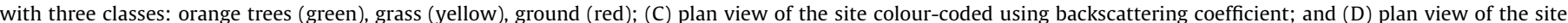

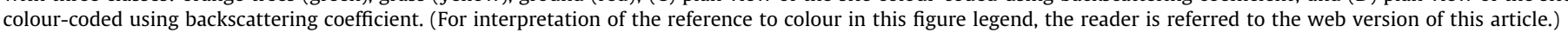


Table 6

Reflectance of three classes based on $\gamma$ of single-peak waveform (SEM-standard error of the mean).

\begin{tabular}{llllll}
\hline Reflectance & Mean & Standard deviation & SEM & Min & Max \\
\hline Ground & 0.60 & 0.051 & 0.0004 & 0.489 & 0.802 \\
Grass & 0.46 & 0.038 & 0.0006 & 0.355 & 0.677 \\
Orange tree & 0.31 & 0.038 & 0.0005 & 0.187 & 0.573 \\
\hline
\end{tabular}

Table 7

Reflectance values for three classes calculated according to waveform class. Standard deviation in brackets.

\begin{tabular}{lllll}
\hline $\begin{array}{l}\text { Class/ } \\
\text { type of } \\
\text { waveform }\end{array}$ & Orange tree & $\begin{array}{l}\text { Orange tree } \\
\text { (from first/ } \\
\text { middle return } \\
\text { of waveforms } \\
\text { with ground } \\
\text { return) }\end{array}$ & Ground & Grass \\
\hline $\begin{array}{c}\text { Single } \\
\text { Two-peak } \\
\text { Three- } \\
\text { peak }\end{array}$ & $0.31( \pm 0.038)$ & - & & \\
\hline
\end{tabular}

differ for single and multi-peak waveforms, where in the latter cases backscattering coefficient $\gamma$ would still depend on the relative relationship between the illuminated areas of the targets involved. Therefore a different procedure is proposed for classification of single and multi-peak waveforms. Three land cover classes (ground, grass and orange trees) were separated on a per point basis using backscattering coefficient $\gamma$ and pulse width alone. Although separation of grass class from ground returns may be ambiguous, a comparison to a different site with no grass class as well as comparison to aerial photography give some confidence that the location of grass returns in the scatterplot is very plausible. The classification accuracy was assessed in two ways; using a combination of elevation and aerial photography it reached $91 \%$. For the classification of single-peak waveforms, a gamma-width scatterplot was used with empirically defined linear relations between $\gamma$ and pulse width to separate three classes. Multi-peak waveforms were first split into two categories (ground and vegetation) based on $\gamma$ of first (or first plus middle) versus last return scatterplots, taking into account the reflectance properties of targets. Subsequently, the grass class was distinguished using a gamma-width scatterplot. Backscattering coefficient $\gamma$ of single-peak waveforms was also used to estimate reflectance of three classes, and these agreed with the published values, giving some confidence in the validity of this approach. Further work will focus on applying this methodology to different vegetation types, as data and ground truth have already been acquired for a number of other tree types. Once this technique is tested on more sites, a way of generalisation and automation will be sought, the spatial distribution of returns and their widths and backscattering coefficients will be used to derive further vegetation structure and density information. This will be validated by comparison to ground truth, and remotely sensed passive microwave and radar of those sites.

\section{Acknowledgments}

This work was funded by Engineering and Physical Sciences Research Council (Grant Number: EP/P505682/1), School of Mathematical and Physical Sciences and School of Systems Engineering of University of Reading. Field campaign data was funded by Australian Research Council Projects LE0560930, DP0557543 and FS100100040. Waveform processing was funded by the National
Centre for Earth Observation. The authors would like to thank Mihai Tanase for the field trip to the orange orchard site, and Professor Victor Becerra for help with the optimisation procedure. Two anonymous reviewers are thanked for their comments and suggestions.

\section{References}

Alexander, C., Tansey, K., Kaduk, J., Holland, D., Tate, N.J., 2010. Backscatter coefficient as an attribute for the classification of full-waveform airborne laser scanning data in urban areas. ISPRS Journal of Photogrammetry and Remote Sensing 65 (5), 423-432.

Bowker, D., Davis, R., Myrick, D., Stacy, K., Jones, W., 1985. Spectral Reflectances of Natural Targets for Use in Remote Sensing Studies. NASA RP-1139.

Bretar, F., Chauve, A., Bailly, J.-S., Mallet, C., Jacome, A., 2009. Terrain surfaces and 3D landcover classification from small footprint full-waveform lidar data: application to badlands. Hydrology and Earth System Sciences 13 (8), 15311544

Briese, C., Hofle, B., Lehner, H., Wagner, W., Pfennigbauer, M., Ullrich, A., 2008. Calibration of Full-Waveform Airborne Laser Scanning Data for Object Classification. SPIE Laser Radar Technology and Applications Orlando, Florida, 19-20 March, p. 8 (on CD-ROM)

Chauve, A., Mallet, C., Bretar, F., Durrieu, S., Pierrot-Deseilligny, M., Puech, W., 2007. Processing full-waveform LiDAR data: modelling raw signals. International Archives of Photogrammetry, Remote Sensing and Spatial Sciences 36 (Part 3/ W52), 102-107.

Ducic, V., Hollaus, M., Ullrich, A., Wagner, W., T., M., 2006. 3D Vegetation Mapping and Classification Using Full-Waveform Laser Scanning. Workshop on 3D Remote Sensing in Forestry, Vienna, Austria, 14-15 February, pp. 222-228.

Gross, H., Jutzi, B., Thoennessen, U., 2007. Segmentation of tree regions using data of a full-waveform laser. International Archives of Photogrammetry, Remote Sensing and Spatial Information Sciences 36 (Part 3/W49A), 57-62.

Guo, L., Chehata, N., Mallet, C., Boukir, S., 2011. Relevance of airborne lidar and multispectral image data for urban scene classification using Random Forests. ISPRS Journal of Photogrammetry and Remote Sensing 66 (1), 56-66.

Heinzel, J., Koch, B., 2011. Exploring full-waveform LiDAR parameters for tree species classification. International Journal of Applied Earth Observation and Geoinformation 13 (1), 152-160.

Hernandez-Marin, S., Wallace, A.M., Gibson, G.J., 2007. Bayesian analysis of lidar signals with multiple returns. IEEE Transactions on Pattern Analysis and Machine Intelligence 29 (12), 2170-2180.

Höfle, B., Hollaus, M., 2010. Urban vegetation detection using high density fullwaveform airborne LiDAR data - combination of object-based image and point cloud analysis. International Archives of Photogrammetry, Remote sensing and Spatial Information Sciences 36 (Part 7B), 281-285.

Höfle, B., Hollaus, M., Hagenauer, J., 2012. Urban vegetation detection using radiometrically calibrated small-footprint full-waveform airborne LiDAR data. ISPRS Journal of Photogrammetry and Remote Sensing 67, 134-147.

Höfle, B., Hollaus, M., Lehner, H., Pfeifer, N., Wagner, W., 2008. Area-based parameterization of forest structure using full-waveform airborne laser scanning data. In: SilviLaser 2008: 8th International Conference on LiDAR Applications in Forest Assessment and Infentory, Edinburgh, UK, 17-19 September, pp. 227-235.

Hofton, M.A., Minster, J.B., Blair, J.B., 2000. Decomposition of laser altimeter waveforms. IEEE Transactions on Geoscience and Remote Sensing 38 (4), 19891996.

Landis, J.R., Koch, G.G., 1977. The measurement of observer agreement for categorical data. Biometrics 33 (1), 159-174.

Lehner, H., Briese, C., 2010. Radiometric calibration of full-waveform airborne laser scanning based on natural surfaces. International Archives of Photogrammetry. Remote Sensing and Spatial Information Sciences 38 (Part 7B), 360-365.

Lin, Y.-C., Mills, J.P., Smith-Voysey, S., 2010. Rigorous pulse detection from fullwaveform airborne laser scanning data. International Journal of Remote Sensing 31 (5), 1303-1324.

Litkey, P., Rönnholm, P., Lumme, J., Liang, X., 2007. Waveform features for tree identification. International Archives of the Photogrammetry, Remote Sensing and Spatial Information Sciences 36 (3/W52), 258-263.

Mallet, C., Bretar, F., 2009. Full-waveform topographic lidar: state-of-the-art. ISPRS Journal of Photogrammetry and Remote Sensing 64 (1), 1-16.

Mallet, C., Bretar, F., Roux, M., Soergel, U., Heipke, C., 2011. Relevance assessment of full-waveform lidar data for urban area classification. ISPRS Journal of Photogrammetry and Remote Sensing 66 (6), S71-S84.

Mallet, C., Lafarge, F., Bretar, F., Soergel, U., Heipke, C., 2009. Lidar waveform modeling using a marked point process. In: 16th IEEE International Conference on Image Processing (ICIP), Cairo, Egypt, 7-10 November, pp. 1713-1716.

Mallet, C., Soergel, U., Bretar, F., 2008. Analysis of full-waveform lidar data for an accurate classification of urban areas. International Archives of Photogrammetry, Remote Sensing and Spatial Information Sciences 37 (Part B3a), 85-92.

McGaughey, R.J., 2012. FUSION Software. <http://forsys.cfr.washington.edu/ (accessed 9.11.12)

Merlin, O., Walker, J.P., Kalma, J.D., Kim, E.J., Hacker, J., Panciera, R., Young, R., Summerell, G., Hornbuckle, J., Hafeez, M., Jackson, T., 2008. The NAFE'06 data 
set: towards soil moisture retrieval at intermediate resolution. Advances in Water Resources 31 (11), 1444-1455.

Monerris, A., Walker, J.P., Panciera, R., Jackson, T., Tanase, M., Gray, D., Ryu, D., 2011. The Third Soil Moisture Active Passive Experiment. Workplan. <http:// www.smapex.monash.edu.au/> (accessed 01.11.12).

Neuenschwander, A., Magruder, L., Gutierrez, R., 2008. Signal processing techniques for feature extraction and classification using small-footprint full-waveform airborne LIDAR. In: IEEE International Geoscience and Remote Sensing Symposium, 7-11 July, pp. III-676-III-679.

Neuenschwander, A.L., Magruder, L.A., Tyler, M., 2009. Landcover classification of small-footprint, full-waveform lidar data. Journal of Applied Remote Sensing, 62.

Persson, A., Söderman, U., Töpel, J.S.A., 2005. Visualization and analysis of fullwaveform airborne laser scanner data. International Archives of Photogrammetry, Remote Sensing and Spatial Information Sciences 36 (Part 3/W19), 103-108.

Reitberger, J., Krzystek, P., Heurich, M., 2006. Full waveform analysis of small footprint airborne laser scanning data in the bavarian forest national park for three species classification. Workshop on 3D Remote Sensing in Forestry, Vienna, Austria, 14-15 February, pp. 229-238.

Reitberger, J., Krzystek, P., Stilla, U., 2008. Analysis of full waveform LIDAR data for the classification of deciduous and coniferous trees. International Journal of Remote Sensing 29 (5), 1407-1431.

Reitberger, J., Schnörr, C., Krzystek, P., Stilla, U., 2009. 3D segmentation of single trees exploiting full waveform LIDAR data. ISPRS Journal of Photogrammetry and Remote Sensing 64 (6), 561-574.

RIEGL, 2012. Riegl <http://www.riegl.com> (accessed 9.11.12).

Roncat, A., Lehner, H., Briese, C., 2011. Laser pulse variations and their influence on radiometric calibration of full-waveform laser scanner data. International
Archives of Photogrammetry, Remote Sensing and Spatial Information Sciences 38 (5/W12), 37-42.

Roncat, A., Wagner, W.T.M., Ullrich, A., 2008. Echo detection and localization in fullwaveform airborne laser scanner data using the averaged square difference function estimator. The Photogrammetric Journal of Finnland 21 (1), 62-75.

Rutzinger, M., Höfle, B., Hollaus, M., Pfeifer, N., 2008. Object-based point cloud analysis of full-waveform airborne laser scanning data for urban vegetation classification. Sensors 8 (8), 4505-4528.

Wagner, W., 2010. Radiometric calibration of small-footprint full-waveform airborne laser scanner measurements: Basic physical concepts. ISPRS Journal of Photogrammetry and Remote Sensing 65 (6), 505-513.

Wagner, W., Hollaus, M., Briese, C., Ducic, V., 2008. 3D vegetation mapping using small-footprint full-waveform airborne laser scanners. International Journal of Remote Sensing 29 (5), 1433-1452.

Wagner, W., Ullrich, A., Ducic, V., Melzer, T., Studnicka, N., 2006. Gaussian decomposition and calibration of a novel small-footprint full-waveform digitising airborne laser scanner. ISPRS Journal of Photogrammetry and Remote Sensing 60 (2), 100-112.

Walker, J.P., Merlin, O., Panciera, R., 2006. National airborne field experiment 2006 workplan. <http://www.nafe.unimelb.edu.au/> (accessed 01.11.2012).

Yao, W., Krzystek, P., Heurich, M., 2012. Tree species classification and estimation of stem volume and DBH based on single tree extraction by exploiting airborne full-waveform LiDAR data. Remote Sensing of Environment 123, 368-380.

Zhu, J., Zhang, Z., Hu, X., 2011. Analysis and application of LiDAR waveform data using a progressive waveform decomposition method. International Archives of Photogrammetry, Remote Sensing and Spatial Information Sciences 38 (5/W12), $31-36$. 